Cahiers $d u$ MONDE RUSSE

\section{Cahiers du monde russe}

Russie - Empire russe - Union soviétique et États indépendants

$50 / 1 \mid 2009$

Écrits personnels. Russie XVIII ${ }^{\mathrm{e}} \mathrm{XX} \mathrm{X}^{\mathrm{e}}$ siècles

\title{
Journaux féminins en langue française, 1780-1850
}

\section{Elena Grecanaja et Catherine Viollet}

\section{OpenEdition \\ Journals}

Édition électronique

URL : https://journals.openedition.org/monderusse/9150

DOI : 10.4000/monderusse. 9150

ISSN : $1777-5388$

Éditeur

Éditions de l'EHESS

Édition imprimée

Date de publication : 31 mars 2009

Pagination : 33-67

ISBN : 978-2-7132-2259-7

ISSN : $1252-6576$

\section{Référence électronique}

Elena Grecanaja et Catherine Viollet, « Journaux féminins en langue française, 1780-1850 », Cahiers du monde russe [En ligne], 50/1 | 2009, mis en ligne le 01 janvier 2009, consulté le 03 septembre 2022 URL : http://journals.openedition.org/monderusse/9150; DOI : https://doi.org/10.4000/monderusse. 9150 


\section{JOURNAUX FÉMININS EN LANGUE FRANÇAISE 1780-1850}

Le corpus que nous analysons est constitué de journaux personnels ${ }^{1}$, pour la plupart manuscrits, se trouvant dans différentes archives russes ${ }^{2}$, datant de la fin du XVIII et des premières décennies du XIX ${ }^{\mathrm{e}}$ siècle. Leur principal point commun est le fait qu'ils ont été rédigés en langue française, par des femmes appartenant à l'aristocratie russe. Il est difficile d'évaluer l'ampleur exacte de ces écrits diaristiques ; les seuls journaux conservés ne constituent probablement que la partie émergée de l'iceberg, un grand nombre d'entre eux ayant très certainement disparu ${ }^{3}$. Parmi les 56 auteurs inventoriés, les femmes, au nombre de 44, prédominent : elles représentent donc presque $80 \%$ des auteurs de journaux en langue française conservés dans les archives ${ }^{4}$.

1. En termes de quantité, il peut s'agir, suivant les auteurs, de quelques pages ou de dizaines de cahiers s'étendant sur plusieurs années.

2. Archives consultées: GARF (Gosudarstvennyj Arhiv Rossijskoj Federacii - Archives d'État de la Fédération de Russie), Moscou; OPI GIM (Otdel pis'mennyh istočnikov gosudarstvennogo istoričeskogo muzeja - Département des manuscrits du Musée historique), Moscou ; IRLI (Institut Russkoj literatury [Puškinskij dom] — Institut de Littérature russe [Maison Pouchkine]), Département des manuscrits, Saint-Pétersbourg ; RGB (Rossijskaja Gosudarstvennaja Biblioteka - Bibliothèque de l'État russe), Département des manuscrits, Moscou ; RGADA (Rossijskij gosudarstvennyj arhiv drevnih aktov - Archives de l'État de Russie des Actes anciens), Moscou ; RGALI (Rossijskij gosudarstvennyj arhiv literatury i iskusstva - Archives de l'État de Russie de littérature et d'art), Moscou.

3. Ainsi, d'après la numérotation des cahiers établie par les diaristes, on peut conclure que quelques journaux d'Elizaveta Šahovskaja des années 1830 ne se sont pas conservés, ainsi que les trois premiers cahiers, de la fin des années 1830, de sa fille Natal'ja. De même, les journaux de Juliette de Krüdener, de 1803, de Marija Bahmeteva, de 1807, et de Sof’ja Murav'eva, de 1841, sont incomplets.

4. Soit environ 120 numéros d'inventaire, textes inédits pour la grande majorité. Quinze femmes diaristes et dix-neuf extraits de leurs journaux sont présentés dans une anthologie récemment parue, où nous proposons un aperçu général de la pratique diaristique féminine de l'époque : Elena Gretchanaia et Catherine Viollet, éds., «Si tu lis jamais ce journal... » : diaristes russes francophones. 1780-1854, P. : CNRS Éditions, 2008. 
En effet, si l'enseignement précoce et l'usage du français, «langue de l'Europe » lettrée, sont partagés à cette époque par tous les membres de l'aristocratie russe, quel que soit leur sexe, les femmes semblent recourir plus volontiers que leurs contemporains masculins à cette langue pour rédiger leur journal personnel, ainsi que leur correspondance.

Qui sont ces diaristes 5 ? La plupart d'entre elles vivent dans les capitales (Moscou et Saint-Pétersbourg), quelques-unes sont d'origine étrangère (allemande). Ce sont des jeunes filles, des femmes mariées, des veuves ou des célibataires ; toutes bénéficient d'une éducation soignée. Leur vie se déroule essentiellement au sein de leur famille, pour certaines aussi à la cour et, malgré certaines ambitions artistiques, elles ne s'adonnent à aucune activité spécifique (mis à part Mme de Krüdener, qui veut devenir, et deviendra un écrivain reconnu). Les journaux de jeunesse s'attachent à décrire les préoccupations, occupations et loisirs de cette classe d'âge : éducation (à domicile), bals, flirts et visites à l'entourage. D'autres journaux sont consacrés aux sentiments pour le (futur) époux, à l'amitié. Un grand nombre d'entre eux relatent des voyages à l'étranger, ou encore la vie quotidienne en Russie. Ils sont imprégnés de morale religieuse, et leurs auteures sont conscientes des contraintes - notamment de bienséance - liées à leur sexe.

Les premiers journaux sont plutôt proches de la chronique familiale, centrés sur des événements d'ordre factuel ; ceux de la période intermédiaire (1795-1820 environ) deviennent plus introspectifs, s'orientent vers les sentiments et le paysage intérieur ; quant à ceux de la dernière période (1820-1854), leur ton est plus varié, ils manifestent une plus grande complexité, une concentration plus marquée sur la personnalité de la diariste, et prennent de la distance, sur le mode ludique ou ironique, vis-à-vis de la perception de soi.

Bien que nous considérions les journaux personnels avant tout comme une pratique d'écriture de soi ${ }^{6}$, il n'en demeure pas moins que ces textes portent en eux de nombreuses informations relevant de l'histoire culturelle. Une histoire culturelle telle qu'elle serait vue et décrite par des individus, « de l'intérieur », au jour le jour, à travers les événements qui tissent la vie quotidienne. En ce sens, ils constituent une source d'information unique en son genre puisque, à la différence de la correspondance, toujours ponctuelle même si elle est abondante, et adressée à un interlocuteur donné, les journaux offrent un récit suivi du vécu des diaristes.

Par ailleurs, le nombre élevé de journaux permet de peindre une fresque aux couleurs subtiles, de relever parallélismes, similitudes et différences. Bien entendu, pas plus que d'autres, aucun des thèmes analysés n'est abordé de manière systématique par les diaristes : c'est au fil des pages manuscrites et des innombrables entrées qu'il faut glaner les informations.

5. Quelques-unes d'entre elles n'ont pu être identifiées, mais leur journal indique qu'elles appartiennent au même milieu.

6. Une parmi d'autres, contemporaines ou non, parfois complémentaires : livres de raison, chroniques familiales, album amicorum, album de citations, mémoires, correspondance... 
Notre approche se propose de caractériser - tout en gardant la richesse des détails - les principaux choix thématiques présents de façon récurrente dans ces journaux, qui attestent de leur cohésion en tant qu'ensemble. Restituer les expériences et activités des diaristes, les espaces matériels et relationnels dans lesquels elles évoluent, les repères culturels qui leur sont propres, c'est saisir leur manière de construire les rapports entre soi et le monde.

Comment, pratiquement, se déroule l'éducation? Quels livres lit-on, et de quelle manière ? Comment se manifeste, concrètement, la relation individuelle à la religion, aux arts ? Quelle place est accordée, dans l'écriture, au corps et à la maladie ? Comment fonctionnent les relations familiales, les réseaux de sociabilité ? Comment se déroulent les nombreux voyages que rapportent les diaristes ? Enfin, qu'apprend-on de leur conception de l'amour, du mariage, des relations avec la gent masculine, des amitiés féminines ?

\section{Une éducation soignée...}

Les journaux - surtout ceux des jeunes diaristes - fournissent de nombreuses informations sur les matières enseignées et les méthodes employées. Assurée à domicile, cette éducation est complétée par de nombreuses lectures et activités culturelles, sans oublier le rôle éminemment formateur des voyages à l'étranger.

Au sein du cercle familial, l'éducation de nos diaristes commence dès leurs plus jeunes années. «Lieu évident et longtemps unique d'éducation pour les femmes, la maison est malheureusement silencieuse pour l'historien. Les apprentissages transmis de mère en fille, de génération en génération, laissent peu de traces tangibles », constatent les spécialistes de l'histoire des femmes ${ }^{7}$. Or les notations contenues, de manière éparse, dans ces journaux permettent de se faire une idée assez précise de la manière dont se déroule l'instruction de leurs auteures.

Elle est généralement assurée sous forme de « leçons » à domicile, soit par des gouvernantes étrangères ou des professeurs, soit par la proche parentèle ${ }^{8}$. Lors d'un voyage à Rome, Sof'ja Aleksandrovna Murav'jeva (1822-1851) se souvient ainsi de sa tante en 1842 : «je me rappellais ma turbulente enfance et les moments

7. Georges Duby, Michelle Perrot, Histoire des femmes en Occident, t. III, $X V I^{e}-X V I I I^{e}$ siècles, sous la dir. de Nathalie Zemon Davis et Arlette Farge, P. : Plon, coll. « Tempus », 1991, p. 142143.

8. Voir notamment : Elena Lihačeva, Materialy dlja istorii ženskogo obrazovanija $v$ Rossii : 1086-1796 [Matériaux pour l'histoire de l'éducation des femmes en Russie: 1086-1796], SPb., 1890 ; J.L. Black, « Educating Women in Eighteenth-Century Russia : Myths and Realities », Canadian Slavonic Studies, 20 (1978), p. 23-42 ; Natal'ja L. Puškareva, Castnaja žizn' russkoj Ženščiny: nevesta, žena, ljubovnica ( $X$-načalo $X I X$ v.) [La vie privée de la femme russe : fiancée, épouse, amante ( $X^{\mathrm{e}}$-début du XIX siècle)], M., Ladomir, 1997, p. 208-220 ; Evelyne Enderlein, «L'éducation des femmes en Russie au XVIII ${ }^{\mathrm{e}}$ siècle », in G. Leduc, éd., L'éducation des femmes en Europe et en Amérique du Nord, de la Renaissance à 1848 : réalités et représentations, P. : L'Harmattan, 1997, p. 349-361 ; O.Ju. Solodjankina, Inostrannye guvernantki v Rossii (vtoraja polovina XVIII-pervaja polovina XIX veka) [Les gouvernantes étrangères en

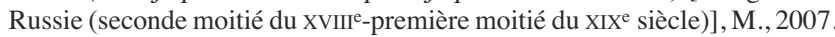


d'ennui que je lui causais lorsqu'elle me donnait les leçons d'histoire Romaine et Grecque $» 9$.

Marija Bahmeteva (dates de vie inconnues), une jeune fille d'environ seize ans, donne un compte rendu précis des leçons d'italien et de guitare que lui donnent régulièrement en 1805 des maîtres italiens ${ }^{10}$. Le programme que suit Ekaterina Aleksandrovna Sojmonova (1811-1879) en 1826 comprend les matières suivantes : histoire ancienne, histoire russe, géographie, traductions de français en anglais, d'allemand en anglais, d'allemand en latin ; danse, dessin, piano, chant, broderie... Un maître français dispense une partie de l'enseignement à Ekaterina et sa fratrie ${ }^{11}$ :

Lundi 2 Janvier 1826. La quantité de mes leçons m'a empêchée depuis le 5 du mois passé a été un obstacle de continuer à écrire mon journal; depuis le 25 Novembre Mr Laforêt a recommencé de nouveau à venir nous donner des leçons $[\ldots] .12$

Âgée de 13 ans, Natal'ja Valentinovna Šahovskaja (1825-1847) relate jour après jour les leçons - qu'elle partage en partie avec ses frères - données par des «maîtres » :

\section{Ce *21 Février 1839 Mardi}

Hier à six heures le maître russe donna sa première leçon, il me plait assez, mais il donne de grandes leçons.

Ce 25 Février 1839 Samedi

Hier à une heure monsieu * Tamis le maître français nous donna sa première leçon, il m'a l'air très doux et très bon, il donne de petites leçons.

Ce premier Mars 1839

Lundi à dix heures le maître russe vint chez nous j'ai été occupée pendant toute la matinée, lorsqu'il s'ennalla je *traduisis pour le maître français qui vient le même jour à 2 heures.

Ce 22 Lundi Mai 1839

Vendredi soir j'ai commencé l'arithmétique avec mon très cher Père, cela ne m'ennuie pas du tout. ${ }^{13}$

C'est avec un monsieur Fenwood, également précepteur de ses frères, qu'elle apprend l'anglais; le piano avec une madame Chicot. La danse de salon est enseignée par un maître de danse, M. Beaulieu, lors de soirées organisées régulièrement chez des cousins.

9. Gretchanaia, Viollet, éds., « Si tu lis jamais ce journal... », p. 311.

10. Ibid., p. 170-186.

11. Les citations respectent l'orthographe et la ponctuation originales de l'auteur, ainsi que les actes scripturaux tels que : bifftre, soulignement, <ajout interlinéaire>. L'astérisque précède les mots dont la lecture est conjecturale. Nos propres interventions (complément d'informations ou de mots abrégés) sont signalées entre crochets droits.

12. Ekaterina Aleksandrovna Sojmonova, Journal de Catiche. Commencé le 9 Août 1825, OPI GIM,f. 395, op. 1, d. 159,1.92v.

13. [Journal, 1838-1840], IRLI, f. 334, d. 977, 1. 43, 45, 46, 76. 
Mais bientôt, pour des raisons financières, on renonce à l'intervention de « maîtres » rémunérés, et l'enseignement se restreint à la seule cellule familiale :

\section{[...] Ce 27 Aout 1839 Dimanche Petersbourg}

Avant hier matin ma très chère Cléopâtre [tante paternelle] me dit que Papa n'a pas assez d'argent pour me donner des maîtres et que par conséquent c'est lui et elle qui me donneront leçons, pour moi je suis très contente de cela puisque j'aime mieux apprendre avec mes parens qu'avec les maîtres $[\ldots]^{14}$.

Une autre diariste contemporaine, Sof ${ }^{\prime} j \mathrm{j} H$. (non identifiée), corrobore ces informations, non sans une certaine impertinence propre à l'adolescence :

\section{Le 25 Février.}

Le matin nous nous occupons avec nos maîtres, j'ai commencé à prendre des leçons de chant chez Rubini et cela m'amuse beaucoup.

Je continue aussi mes leçons de littérature avec l'insupportable Mr Ruault, c'est la plus ridicule figure que j'ai vu depuis longtemps. Une tête longue d'une aune posée sur un petit corps disgracieux et maigre et ce petit corps monté sur deux fluttes. - Je ne puis le regarder sans rire. - Surtout quand il déclame dans le sol.[iloque] de Rodrigue. - Sans le vouloir je sens que je suis impertinente avec lui. - Laure peut en dire autant. ${ }^{15}$

Quant à la toute jeune Ekaterina Aleksandrovna Saburova (épouse Fredericks ; 1832-1917), âgée de dix ans, c'est en ces termes qu'elle relate le bilan de sa journée :

J'ai bien su toutes mes leçons excepté l'anglais que j'aurais pu mieux savoir. De la musique [piano] j'ai été contente. Je me suis fâchée de ce que Melle Clark m'a dit de ne pas répéter la leçon russe. Après diner j'ai eu de l'impatience à la leçon d'arithmétique. [entrée du 11 août 1842] ${ }^{16}$

Ce journal et d'autres montrent à quel point l'instruction proprement dite est étroitement liée à l'apprentissage des bonnes manières, au «savoir-vivre » que l'on attend des jeunes filles.

Le programme d'études que partagent, dans ses grandes lignes, la plupart des contemporaines est principalement axé sur les langues vivantes étrangères - mais on apprend aussi le russe - , et les arts d'agrément. On ne se contente pas d'un savoir livresque : la pratique orale des langues étrangères se double de nombreuses lectures dans les langues étudiées, et d'exercices de traduction.

Les noms des «maîtres » et gouvernantes, généralement d'origine étrangère, émaillent le texte des journaux - qui ne donnent que peu de renseignements sur les

14. Ibid., 1. 95 .

15. Sof'ja H., [Journal, 1839-1840] (classé anonyme ; cette diariste n'a pu être identifiée), RGALI, f. 1337, op. 1, d. 332, 1. 60.

16. Gretchanaia, Viollet, éds., « Si tu lis jamais ce journal... », p. 322. 
modalités de leur recrutement, sauf dans un cas, qui concerne les frères (plus jeunes) de Natal’ja Šahovskaja :

Marseille, 1841. Mardi matin nous vîmes arriver chez nous Monsieur Amedée Bechard, que Duplan nous envoyait pour professeur pour nos garçons, nous l'en avions prié à Gréoulx et il remplit parfaitement sa commission. Monsieur Bechard nous plut assez au premier abord, mais il nous effraya cependant en nous disant qu'il ne s'était jamais occupé d'enfants, malgré cela il remplit parfaitement ce nouvel et triste état qu'il a embrassé, il est partit pour la Russie avec Papa et Maman et on nous écrit qu'il est un excellent professeur et menin [... $]^{17}$.

L'histoire - en particulier l'histoire ancienne, mais aussi celle des pays d'Europe et de la Russie - y tient une grande place et semble être une matière extrêmement appréciée, considérée comme essentielle ; l'enseignement en est étayé par de nombreuses lectures.

Principes moraux et religieux sont généralement enseignés par la parenté, et étayées par des lectures pieuses : c'est un rôle dévolu en priorité à la mère, ainsi qu'aux autres femmes de l'entourage.

Quant à la musique, qui semble être le plus souvent enseignée par des professeurs italiens, le chant recueille tous les suffrages ; viennent ensuite clavecin ou piano, guitare, harpe. Plusieurs diaristes mentionnent également des cours de dessin et de peinture.

$\mathrm{Au}$ fil des journaux, on apprend que ces jeunes filles exercent des activités sportives, principalement l'équitation - de manière parfois téméraire, ainsi qu'en témoignent quelques récits ${ }^{18}$. Natal' ja Petrovna Golicyna (1744-1837) livre un récit détaillé de ce qui semble être un véritable exploit sportif (elle est alors âgée de 22 ans) :

$\mathrm{Au}$ mois de Juen et de Julliet de lannee 1766 il y eu un grand Carousel qui consistoit en 4: quadrilles [...], les Dames étoit assise dans les chars superbe, [...] pour gagner le premier prix il faloit faire les poins que je vai menssionnée, cassé la Lance au premier, tiré un coup de Pistollet entre les oreille d'un Ours, jeter le javelot pour qu'il s'arrete sur la langue du Lion, levé le quasque de dessu un petit pied destal, coupé la tête au hidre, et courire l'anneaux. J'eu le bonheur de gagner les deux fois le premier prix. ${ }^{19}$

Certaines diaristes font état d'autres matières, moins connotées comme spécifiquement féminines, et qui font généralement partie de l'éducation masculine ${ }^{20}$ :

17. [Journal, 1841-1842], RGALI, f. 1337, op. 1, d. 292,1. 29.

18. Voir par exemple Gretchanaia, Viollet, éds., « Si tu lis jamais ce journal... », p. 58.

19. Ibid., p. 82-83. Le premier prix de cet exploit est un diadème orné de diamants.

20. Telles que latin, algèbre, physique expérimentale, physiologie, logique, philosophie, architecture civile et militaire, droit et jurisprudence, histoire des constitutions et gouvernements, politique - matières destinées à préparer à une carrière militaire ou diplomatique. Voir par exemple A. Woronzoff-Dachkoff, C. Le Gouis, C. Woronzoff-Dachkoff, éds., Princesse Dachkova, Mon Histoire : Mémoires d'une femme de lettres russe à l'époque des Lumières, suivis des lettres de l'impératrice Catherine II, P. : L'Harmattan, 1999. 
arithmétique, géométrie, physique, astronomie, botanique - dont elles acquièrent au moins des notions.

Pour soignée qu'elle soit, l'éducation proposée à ces jeunes filles nobles a cependant ses limites ; certaines d'entre elles s'en montrent parfaitement conscientes. Pourtant la soif de s'instruire, liée à des ambitions littéraires ou artistiques, semble vive chez certaines :

J'aime tant le chant, c'est une passion malheureuse que j'ai là, car ayant de la voix et pouvant m'appliquer le plus que possible, ne m'ennuyant jamais d'exercer, je n'ai cependant pas les moyens d'apprendre à chanter comme je l'aurais désiré, plusieurs années de suite sans discontinuer, mais il faudrait pour cela rester en Italie..$^{21}$

Ou encore, à propos des ambitions littéraires :

Mardi 12 [août 1805]. Après le déjeuner j'ai commencé à lire Mme Genlis. Mon admiration pour cette femme savante s'augmente de jour en jour. Quel style enchanteur ! Quelles pensées profondes! Quels sentiments ! Elle fait beaucoup d'honneur à notre sexe. J'envie ses talents. J'aurois voulu être son écolière.22

Reste à souligner le rôle éducatif que joue le journal lui-même, dont la pratique semble être souvent sollicitée, ou tout au moins conseillée, et s'exerce parfois sous surveillance : à la fois atelier d'écriture, discipline quotidienne, guide spirituel, mise en mémoire du vécu, lieu d'expression des sentiments... et prétexte à la formation du style, d'après les modèles littéraires. Ces journaux portent d'ailleurs directement trace des fruits de l'enseignement, en particulier de l'aisance à manipuler les langues étrangères (anglais, allemand, italien) ainsi que le russe, puisqu'ils comportent des passages rédigés dans ces langues.

L'éducation se poursuit parfois à l'âge adulte. Ainsi la princesse Elizaveta Aleksandrovna Šahovskaja (née Muhanova ; 1803-1836) relate-t-elle dans son journal ses efforts pour apprendre les sciences exactes, enseignées par son mari Valentin à ses sœurs et elle-même :

Valentin a proposé d'apprendre chaqu'après dinner la géométrie comme une préparation à l'Astronomie - la première leçon m'a paru si ennuyeuse, si difficile [...]. L'année [18]28 et 29, j'avois bien pu faire un cours de phisique, un autre de philosophie, et écouter pour récréation les romans de Walter Scott ; et maintenant pour mes 30 ans révolus me voilà incapable de prendre le dessus sur moi. - Je me plais à croire que je comprendrai mieux l'Astronomie et qu'elle m'intéressera davantage. ${ }^{23}$

21. Praskov’ja Mihajlovna Golynskaja (1822-1892), [Journal, 1841-1842], RGB, f. 336/II, carton 78, d. $2,1.35$.

22. Gretchanaia, Viollet, éds., « Si tu lis jamais ce journal... », p. 179.

23. Elizaveta Šahovskaja, Journal. Commencé à Colp le 15 mars 1832, RGB, f. 336/2, carton $47, \mathrm{n}^{\circ} 5,1.27-27 \mathrm{v}$. 


\section{... et cosmopolite}

La plupart de ces jeunes filles voyagent fréquemment à travers l'Europe, et pour des périodes prolongées d'un à deux ans - ainsi qu'en témoignent de nombreux journaux. Si ces voyages ne sont pas comparables au Grand Tour ${ }^{24}$, privilège de leurs frères, l'une de leurs principales raisons d'être (à côté de motifs d'ordre familial ou médical) est qu'ils constituent l'occasion de perfectionner leur éducation, et de favoriser la rencontre avec des cultures étrangères.

Ainsi l'éducation se poursuit-elle au gré des séjours et déplacements: nos jeunes diaristes bénéficient alors de cours de langue et de littérature, de dessin, de musique (notamment à Pise et à Naples, avec des maîtres italiens), d'équitation, prodigués par des professeurs autochtones. Parallèlement, elles mènent une existence sociale et mondaine fort active, s'intégrant à la vie culturelle locale, et sont invitées à d'innombrables bals et réjouissances. Très consciencieusement, elles visitent musées et monuments, cabinets de curiosités, jardins et bibliothèques, églises et autres lieux de culte ${ }^{25}$.

De temps à autre, elles assistent à des conférences, et visitent des lieux plus surprenants : navires, bagne (à Toulon), asile d'aliénés ; à Paris, elles participent à des séances de magnétisme et de « somnambulisme ». On va très fréquemment au spectacle, dans chacune des villes visitées : théâtre, ballet, opéra, opéra-comique. Sont alors longuement commentés par des spectatrices exigeantes, de manière enthousiaste ou incisive, les performances et mérites respectifs des interprètes.

Les journaux de voyage contiennent nombre de comparaisons et de jugements témoignant de la rencontre des cultures. Une certaine « acculturation » préalable se conjugue fréquemment avec une véritable curiosité, fort éclectique au demeurant, et une ouverture d'esprit souvent remarquable.

À travers l'apprentissage des langues, les lectures, les spectacles, ou grâce aux voyages de formation, l'éducation de ces jeunes diaristes s'inscrit d'emblée dans une dimension européenne et cosmopolite. Cependant, destinées avant tout au mariage, à l'éducation des enfants et à la transmission de ces valeurs culturelles au sein de la société, certaines d'entre elles regrettent de ne pouvoir tirer un meilleur parti de cette éducation soignée et du développement de leurs talents : elles rêvent de devenir cantatrice, peintre, traductrice, écrivain... 


\section{Lectures}

La lecture est une des occupations favorites, qui trouve sa place dans les journaux sous forme de citations ou de mention des livres lus, accompagnée parfois par des commentaires.

Dans les tout premiers journaux des années 1780 - début des années 1790, journaux de voyage pour la plupart, les lectures ne sont pratiquement pas mentionnées. Ces journaux ont essentiellement pour but de fixer des impressions liées au monde extérieur, qui se succèdent rapidement. Ces premières diaristes évitent en général de parler de leur vie personnelle, de leur monde intérieur, qui se trouve à cette époque de plus en plus mis en relation avec les lectures.

Cette activité occupe en revanche une place importante dans les journaux plus tardifs, à partir de la fin des années 1790, qui rendent compte de la vie quotidienne et tendent, notamment grâce à la vogue de la sensibilité, vers une plus grande intériorisation. Par ailleurs, la pratique alors courante de tenir cahiers et albums, où l'on transcrit des passages de livres lus qui servent de repères moraux ou font écho aux sentiments éprouvés, contribue évidemment à réduire le compte rendu des lectures dans les journaux personnels. Ainsi une jeune fille, Praskov’ja Vasil’evna Naryškina (dates de vie inconnues), mentionne peu ses lectures dans son journal de 1811, lectures auxquelles est pourtant consacré son album de la même époque ${ }^{26}$. Les cahiers de citations des années 1820-1830 de la princesse Elizaveta Aleksandrovna Šahovskaja donnent un tableau des livres lus beaucoup plus vaste que ne le laissent supposer ses journaux de l'époque 27 .

Les titres et les auteurs mentionnés dans les journaux, ainsi que les citations, reflètent le degré de diffusion des différents genres et œuvres littéraires dans les milieux cultivés de la Russie. Dans la plupart des journaux figurent des ouvrages français ou traduits en français.

La liste des Livres envoyés à la campagne l'année 1797, inscrite au début du journal, datant de cette époque, d'une jeune fille, Ekaterina Petrovna Čirikova (épouse Kvašnina-Samarina, morte en 1836) inclut, à part les ouvrages nouvellement parus ou réédités d'auteurs français (Mme de Genlis, Mme de Staël, Florian, Ducray-Duminil, Roucher, Vernes-de-Luze, Dupaty), un nombre de romans, dans le genre gothique, traduits de l'anglais, ainsi que la traduction française du Voyage sentimental de Sterne. Le roman sentimental et gothique domine au sein de cette liste, conformément aux goûts de l'époque. Comportant 38 titres, sa liste inclut cependant deux livres en russe : les œuvres de Vasilij Kapnist et la traduction par Nikolaj Karamzin des nouvelles de Marmontel. Dans son journal, Ekaterina Čirikova admire les « maîtres de la sensibilité » - Florian, Gessner et Karamzin ; elle transcrit des passages des Rêveries du promeneur solitaire de Jean-Jacques

26. Praskov’ja Naryškina, Album et Journal, RGADA, f. 1272, op. 1, d. 134-135.

27. RGB, f. 336/II, carton 46, d. 24 ; carton 70, d. 4. 
Rousseau et du roman de Mme de Genlis, Les chevaliers du Cygne (1795), qu'elle met en relation avec ses états d'âme ${ }^{28}$.

Les journaux russes de la fin XVIII - première moitié du XIX siècle attestent surtout la vogue durable des romans féminins français orientés vers le modèle de la littérature «sensible». En 1805, Marija Bahmeteva, qui choisit comme épigraphe une citation tirée de la Nouvelle Héloïse, loue avec effusion les œuvres de Mme de Genlis ${ }^{29}$.

Eugène de Rothelin (1808), le roman de Mme de Souza, est mentionné dans le journal de 1828 de Varvara Sergeevna Suhotina (née Domašneva ; vers 1795-après 1856) qui en fait la lecture lors de son voyage à l'intérieur de la Russie.

Anastasija Ivanovna Kolečiskaja (née Lykošina; 1800-1871) relit Valérie (1803) de Mme de Krüdener en 1841, qualifie cet ouvrage de «roman délicieux ${ }^{30}$, et en transcrit certains passages ${ }^{31}$. La lecture est considérée comme un événement majeur par cette diariste, qui réserve dans ses entrées une large place à des citations et à ses propres commentaires. Les titres des ouvrages qu'elle a lus sont d'ailleurs mentionnés dans la table des matières de son journal.

Quant à Mme de Staël, ce sont ses ouvrages non fictionnels qui sont mentionnés dans les journaux féminins de l'époque, ses romans étant apparemment considérés comme une lecture peu convenable pour des femmes, a fortiori pour des jeunes filles. Ce n'est qu'en 1825, après son mariage, qu'Elizaveta Šahovskaja lira Delphine ${ }^{32}$. Auparavant, en 1820, elle cite, dans un de ses cahiers de citations, des passages du traité de Mme de Staël De l'influence des passions sur le bonheur des individus et des nations ${ }^{33}$, et dans la liste de livres qu'elle a lus figure De l'Allemagne ${ }^{34}$. En cette même année 1820, Anna Petrovna Kern (née Poltorackaja ; 18001879) transcrit dans son journal des passages de ce dernier ouvrage, passages qu'elle s'applique souvent à elle-même ${ }^{35}$. La comtesse Marija Jur'evna Tolstaja (née princesse Obolenskaja ; 1802-après 1847) en mentionne également la lecture en $1822^{36}$.

28. IRLI, f. 416, d. 1, 1. 32v, 84v-86.

29. Gretchanaia, Viollet, éds., « Si tu lis jamais ce journal... », p. 179.

30. RGALI, f. 427, op. 1, d. 157,1. 36 .

31. En 1820, un jeune officier, le prince Valentin Mihajlovič Šahovskoj (1801-1850) lit uniquement, lors d'un voyage vers le sud de la Russie, des romans en français écrits par des femmes : Malvina (1801) de Mme Cottin, Les Vœux téméraires, ou l'Enthousiasme (1799) de Mme de Genlis et Saint Clair des Isles, ou Les exilés à l'île de Barra (1808), traduction libre par Mme de Montolieu du roman anglais d'Elisabeth Helme (RGB, f. 336/II, carton 63, d. 24). Dans son journal tenu en 1823 à Moscou, Valentin dit avoir lu à ses sœurs Amélie Mansfield (1803), roman de Mme Cottin (Ibid., 1. 12).

32. RGB, f. 336/II, carton 47, d. 11,1.1.

33. Ibid., carton 70, d. 1,1. 2, 10v-11.

34. Ibid., d. 5, 1. 7 .

35. A.P. Kern (Markova-Vinogradskaja), Vospominanija. Dnevniki. Perepiska [Souvenirs. Journaux. Correspondance], M. : Hudlit, 1974.

36. RGADA, f. 1280, op. 1, d. 134,1. 44. 
Plus tard, en 1841, c'est une tante qui fait la lecture de Corinne à ses jeunes nièces, alors qu'elles se trouvent en Italie ${ }^{37}$.

Les lectures des femmes sont donc plutôt « sages », conformément à leur image telle qu'elle se construit dans les journaux : aucun titre qui pourrait paraître « douteux » n'y apparaît. Les romans y sont mentionnés plus rarement que les lectures sérieuses - en premier lieu des ouvrages historiques, philosophiques, critiques, utiles avant tout à l'instruction : parmi les auteurs mentionnés, on trouve notamment Montesquieu, Gibbon, La Harpe, Condillac, Mme Campan, Mignet, Guizot $^{38}$.

Si les lectures russes ne tiennent généralement pas une grande place dans les journaux rédigés en français, elles sont cependant présentes, et montrent notamment que les femmes en Russie maîtrisent à cette époque la langue de leur pays . Ainsi, Ekaterina Čirikova donne une traduction (qui paraît être de sa main) en français du poème russe de Nikolaj Karamzin publié dans l'almanach Aglaja $^{39}$, ce qui prouve qu'elle lit les périodiques russes de l'époque. Elizaveta Šahovskaja déclare, dans son journal de 1820, qu'elle lit l'Évangile en russe et en slavon ; en outre, ses listes des livres lus comportent des titres d'ouvrages en russe ${ }^{40}$.

Les lectures spirituelles portent, dans les journaux du premier quart du XIXe siècle, l'empreinte de l'intérêt de la société russe pour l'expérience religieuse occidentale, intérêt qui atteint son apogée lors du règne d'Alexandre Irer, mais se maintient les années suivantes. Les diaristes apprécient des auteurs tant catholiques que protestants, des ouvrages piétistes et maçonniques. L'Imitation de Jésus-Christ, les lettres spirituelles de Fénelon, les œuvres de saint François de Sales et de Massillon sont parmi les plus lues.

Praskov'ja Naryškina, dans son journal de 1811, note : « Je ne suis en Paix avec moi-même que lorsque je lis quelques chapitres de l'Imitation ou quelques Lettres de Fénelon $»^{41}$.

Elizaveta Šahovskaja, à part l'Imitation de Jésus-Christ et la traduction française du Combat spirituel de Lorenzo Scrupoli, livres de chevet des catholiques, mentionne dans son journal des ouvrages maçonniques, comme La connaissance de soi-même de John Mason (1783), qu'elle lit en anglais ${ }^{42}$, et La Vérité de la religion en général de Gotthard Friedrich Stender (1785, rééd. 1820), qu'elle lit dans la traduction russe ${ }^{43}$.

37. Voir Gretchanaia, Viollet, éds., « Si tu lis jamais ce journal... », p. 315-316.

38. Ce genre de lecture semble être partagé par d'autres femmes européennes contemporaines, voir par exemple le Journal de Magdalena van Schinne, 1786-1805, textes présentés et recueillis par Rudolf Dekker et Anje Dik, P. : Côté-Femmes, 1994, p. 25.

39. Voir Gretchanaia, Viollet, éds., « Si tu lis jamais ce journal... », p. 137-138.

40. RGB, f. 336/II, carton 47, d. 11 ; carton 70, d. 1, 5.

41. RGADA, f. 1272, op. 1, d. 134,1. 5.

42. RGB, f. 336/2, carton 47, d. 2, 1. 54v.

43. GARF, f. 1738, op. 1, d. 8,1.15v. 
Varvara Suhotina, lors de son voyage de 1828, découvre « un superbe ouvrage », De l'importance des opinions religieuses (1788) de Jacques Necker, en transcrit des passages et conclut : "Cet ouvrage est si beau qu'il faudrait le copier d'un bout à l'autre, et le savoir par cœur $»^{44}$.

Les journaux de la fille d'Elizaveta Šahovskaja, Natal'ja, et de sa cousine Sof'ja Murav'eva témoignent du fait que ces deux jeunes filles et leur entourage familial portent, au début des années 1840, un grand intérêt aux œuvres d'Emmanuel Swedenborg qu'elles lisent en traduction française ${ }^{45}$.

\section{Pratiques religieuses}

La plupart des femmes diaristes sont formées au sein de l'Église orthodoxe russe. Le compte rendu des pratiques religieuses auxquelles elles assistent (liturgies qu'elles désignent par «messes ») n'occupe pas beaucoup de place dans les journaux et s'inscrit dans le cadre d'autres occupations quotidiennes. Ces descriptions se font plus détaillées lors des pèlerinages à l'intérieur de la Russie ou des grandes fêtes. Marija Tolstaja, dans son journal de 1823, décrit en détail les offices de la Semaine sainte, qui rassemblent la haute société de Saint-Pétersbourg. Anastasija Kolečickaja consacre, dans son journal de 1841, plusieurs pages à son séjour au monastère des grottes de Kiev, exprimant son admiration et ses sentiments pieux. La composante religieuse est souvent liée, dans les journaux des femmes, à leur vie affective. Pour Marija Bahmeteva, la religion est avant tout le moyen de créer un lien sentimental supplémentaire entre elle et son bien-aimé (à qui est adressé son journal) lors de leur séparation :

J'ai passée la matinée à méditer, à rêver et à songer à toi, mais maman m'a appelée pour aller à l'église avec elle, j’y ai été, j'ai porté mes vœux fervents au Ciel pour votre conservation ${ }^{46}$.

Lorsqu'après son mariage, Marija se sent victime de son amour, et subit les tourments de ce qu'elle perçoit comme l'indifférence de son mari, le discours religieux disparaît pratiquement de son journal. Elle n'est préoccupée que par la conduite de son mari envers elle, obsédée par l'idée qu'il a cessé de l'aimer. Elle a «l'enfer dans le cœur $»^{47}$, et ses prières se transforment en un cri de désespoir :

Après le dîner je suis allée avec maman à l'église, j'y ai tant prié Dieu que maman même s'était étonnée, j'ai prié le très haut de me rendre mon bonheur passé ou de finir mon existence ${ }^{48}$.

44. RGADA, f. 1280 , op. 1, d. 136,1. 18v-19.

45. Voir Gretchanaia, Viollet, éds., « Si tu lis jamais ce journal... », p. 294, 309.

46. RGADA, f. 1256, op. 1, d. 1153,1. 17.

47. Ibid., 1. 66.

48. Ibid., 1. 67. 
L'Église ne lui procure aucun réconfort, elle se sent parfois « forcée » d'assister aux offices divins ; la femme d'un prêtre, venue en visite, l'importune.

La tendance à associer discours religieux et sentimental est une des caractéristiques du journal d'Elizaveta Šahovskaja. De nombreuses prières adressées à Dieu concernent exclusivement son bien-aimé, le prince Valentin Šahovskoj, à qui sont consacrés plusieurs de ses journaux de jeune fille :

Hier en me couchant, j'ai prié Dieu pour toi, comme je le fais tous les matins et soirs, je l'ai conjuré de veiller sur toi, de t'accorder un bonheur parfait. Ah ! je l'ai aussi prié que Valentin m'aime, qu'il ne m'oublie pas...49

Je te rends grâce, oh mon Dieu, pour ces jouissances que tu m'accordes, la certitude d'être aimée est la plus grande des félicités. Valentin et moi, nous nous plaignons souvent quand nous sommes ensemble que nous ne pouvons l'être constamment. Mon Dieu, que ces plaintes ne nous fassent mériter la privation de notre bonheur, de grâce ne faites attention qu'à notre reconnaissance ${ }^{50}$.

Lors de leurs voyages en Europe, les diaristes du XVIII ${ }^{\mathrm{e}}$-premier quart du XIX ${ }^{\mathrm{e}}$ siècle peuvent faire tout naturellement leurs dévotions dans des églises catholiques. C'est le cas de la baronne Natal'ja Mihajlovna Stroganova (née princesse Beloselskaja ; 1743-1819) en 1780, à Bruxelles, et de Ekaterina Ivanovna Vadkovskaja (née comtesse Černyševa ; 1765-après 1826) en 1787, à Rome ${ }^{51}$. Natal'ja Ivanovna Kurakina (née Golovina, 1766-1832) note, dans son journal de 1816, en route vers Lausanne, qu'elle a entendu la «messe» catholique «avec la même ferveur presque » que si elle était à «une messe grecque », et y a remarqué «peu de différence $\gg^{52}$.

Dans les journaux des années 1840, apparaît une résistance certaine face aux pratiques catholiques. Sof 'ja Murav'eva et Elizaveta Vasil'evna Davydova (1823-1902) critiquent les offices qu'elles observent en Italie, notamment les visites au Pape ${ }^{53}$. Cependant ce regard critique n'empêche pas les diaristes de fréquenter assidûment les églises catholiques et d'en donner des descriptions détaillées.

L'intérêt pour les pratiques religieuses autres que catholiques, et parfois autres que chrétiennes, se traduit dans la fréquentation, lors des voyages, de lieux de culte protestant et juif. La comtesse Anna Ivanovna Tolstaja (née princesse Barjatinskaja ; 1770-1825) raconte, dans son journal de 1790, sa visite en Allemagne d'un « site des

49. RGB, f. 336/2, carton 47, d. $2,1.9$.

50. Ibid., d. 3,1. 1 .

51. Zapiski baronessy N.M. Stroganovoj [Notes de la baronne N.M. Stroganova], Russkij bibliofil, n 4, 1914, p. 34 ; Journal de Ekaterina Vadkovskaja, OPI GIM, f. 272, op. 1, d. 3, 1. 110 .

52. N.I. Kourakina, « Souvenirs », in Fiodor Kurakin et Vladimir Smoljaninov, éds., Devjatnadcatyj vek. Istoričeskij sbornik [Dix-neuvième siècle. Recueil historique], vol. I, M., 1903, p. 139.

53. Journal de Sof' ja Murav'eva, RGB, f. 336/2, carton 78, d. 3, 1. 19v ; « Journal de Elizaveta Davydova » in Gretchanaia, Viollet, éds., « Si tu lis jamais ce journal... », p. 268. 
Hernhütter ${ }^{54}$, une secte piétiste, et en Hollande, de la même «société des Hernhütter », d'une « église luthérienne » et d'une synagogue ${ }^{55}$. Natal'ja Šahovskaja assiste en France, avec toute sa famille, aux assemblées de l'Église de la Nouvelle Jérusalem, secte protestante fondée sur les œuvres de Swedenborg.

\section{Du corps et des maladies}

La description de l'état physique est un thème qui semble être autorisé dans les journaux personnels ; il apparaît déjà dans le Journal de voyage de Montaigne (1580-1581), publié en 1774. Au XVIII e siècle, la description de l'état physique se fait plus fréquemment que celle de l'état moral, et souvent en tient lieu. Ainsi les premières diaristes, telles que la baronne Natal'ja Mihajlovna Stroganova ou la princesse Ekaterina Petrovna Barjatinskaja (née princesse Holstein-Beck ; 17501811 ) insistent-elles sur leurs maux physiques afin de traduire les sensations de tristesse qui accompagnent leur départ, après la séparation d'avec leurs proches ${ }^{56}$.

Le discours sur le corps s'oriente dans deux directions : les plaisirs (essentiellement bals et promenades) et le corps souffrant. Si les plaisirs n'occupent guère de place, les diaristes parlent abondamment des maux divers qui les assaillent, ainsi que leur entourage, et des manières de les soigner.

Attaquée, depuis environs dix mois, à St. Petersbourg, d'une maladie rheumatique qui m'avoit réduit dans un état à ne pouvoir faire usage de mes membres qu'avec des douleurs aigües, les médecins me conseillèrent d'aller prendre les Bains d'Aix-la-Chapelle. ${ }^{57}$

C'est en ces termes que Ekaterina Barjatinskaja, âgée d'une quarantaine d'années, entreprend son journal (1789-1798). Voyageant en 1830 avec sa mère aux environs de Moscou, Olga Ivanovna Orlova-Davydova (née princesse Barjatinskaja ; 1814$1876)^{58}$ est retenue en quarantaine et déplore de ne pouvoir poursuivre son voyage en raison de la pandémie de choléra-morbus ${ }^{59}$ qui sévit alors dans toute la région. Sa mère l'occupe à fabriquer « des petits sachets de chlore », qui seront distribués en guise de protection dans les villages environnants. Elizaveta Vasil'eva, quittant

54. [Journal], RGB, f. 301, carton 1, d. 22,1.32v.

55. Ibid., 1. 35-35v. Ces visites sont proches de celles que fait en Hollande aussi, à la même époque, en 1794, Amable-Emilie de Châtillon, duchesse d'Uzès, qui décrit dans son récit de voyage le culte grec qui l'a «fort intéressé », les assemblées de Quakers et de «luthériens ». (Duchesse d'Uzès, « Récit de voyage depuis mon départ de France en 1790 », Archives nationales, 265 AP 542).

56. Voir Gretchanaia, Viollet, éds., « Si tu lis jamais ce journal... », p. 74, 105-106.

57. Ibid., p. 105.

58. Olga Orlova-Davydova, «Ce qui s'est passé de plus remarquable pour moi depuis notre retour de Kamenka le 23 septembre », RGB, f. 219, carton 92, d. 2.

59. Il s'agit de la deuxième pandémie de choléra qui, partie d'Asie, s'étendit à toute l'Europe (1826-1841). 
Odessa en 1836, se rend en Italie en bateau, par Constantinople et la Grèce : elle se plaint amèrement des semaines passées en quarantaine (en raison de la peste) avant d'être autorisée à poursuivre son voyage :

Dimanche. Le 11 Octobre. [1836] [Trieste]. Ce matin nous avons quitté le bâtiment nous ferons quarantaine dans le lazaret St Charles. Nous avons dû nous montrer au médecin, ainsi que les matelots qui devaient se donner des coups aux genoux et sous les bras pour prouver qu'ils n'ont pas la peste, ni aucun symptôme de cette maladie, c'était très drôle de les voir.

Jeudi. Le 15 Octobre. J'aime un million de fois mieux notre quarantaine d'Athènes que celle-ci; là je voyais toujours quelqu'un, ici personne, nous sommes dans une vraie prison entourées de murailles et enfermées de tous côtés. La Comtesse et moi nous voulions sortir un peu, eh! bien le gardien nous a menées au cimetière de la quarantaine $[. .$.$] ; c'est terrible, depuis j'ai toujours$ des morts devant moi. ${ }^{60}$

D'autres maux plus ordinaires sont fréquemment mentionnés dans les journaux : maux de dents (arrachées par le dentiste local), invasion de moustiques, abcès, coups de soleil, indigestions, refroidissements, grippes, rhumatismes, « maux de nerfs ». En Italie, où les pièces ne sont guère chauffées en hiver, on souffre du froid et de l'humidité : « [À Pise] Comme il faisait froid alors dans nos chambres nous nous transportâmes toutes dans deux chambres $»^{61}$.

Au cours des longs voyages en Europe, indispositions, malaises, infections font partie du quotidien :

Ce 5 Aout/24 Juillet [1842]. De nouveau j'ai été malade ces jours-ci à cause de mes pieds et de la fièvre. [...] J'espère que je suis tout à fait remise, aussi maintenant tout le reste de notre petite société est malade, Cléopâtre a une fluxion, Lilia a une forte fièvre, Catiche a commencé à avoir hier soir un malaise, Sophie a été très souffrante tous ces jours. [...] Il n'y a que Pauline, qui tousse pourtant, qui soit sur pieds à soigner les malades. ${ }^{62}$

Afin de se fortifier, certaines diaristes font des cures de raisin, et prennent des bains de mer quotidiens lorsqu'elles se trouvent dans le sud de la France ou en Italie. Pour un mal de tête, il arrive qu'on pose des sangsues et pratique des saignées ${ }^{63}$. Les déplacements par bateau (goélettes ou pyroscaphes) sont prétexte à des descriptions, souvent pittoresques, du mal de mer :

Le 19 Juillet 1841 Samedi. À peine le bateau à vapeur commença à voguer, Cléopâtre eut le mal de mer, moi craignant de l'avoir aussi je me dépêchais d'aller me coucher en voiture avec Maman [...] qu'est-ce qui peut se comparer au mal de mer c'est bien la maladie la plus ennuyeuse, pour ne pas l'avoir il

60. Elizaveta Vasil’eva, [Journal, 1836-1837], RGALI, f.1337, op. 1, d. 24,1. 27v-28v.

61. Natal’ja Šahovskaja, [Journal, 1841-1842], 1. 32.

62. Ibid.,1. 88v.

63. Olga Orlova-Davydova, « Ce qui s'est passé... », 1. 6-7. 
fallait rester coucher tout le temps cela ne m'arrangeait pas beaucoup aussi je ne le fis pas et j'ai vomis une quantité de fois [...]

À la vue du bateau à vapeur il nous semblait avoir le mal de mer, nous allâmes avec un courage tout à fait héroïque à la rencontre de notre petite torture car le mal de mer est je suis sûre dans le genre des tortures. ${ }^{64}$

Enfin on a levé l'ancre et nous nous vîmes bientôt voguant au milieu de la mer ; j'étais enchantée de tout ce que je voyais [...]. Mais peu à peu le mouvement devenait plus fort les vagues nous jetaient tantôt en haut tantôt en bas et au bout d'une bonne vingtaine de minutes nous fûmes si joliment ballotés que nous pouvions à peine nous tenir sur pied ; [...] au commencement c'était très drôle mais hélas ! la tragédie suivit de près la comédie. Catia la première descendit dans la Cabine ; car elle sentait que si elle restait un moment de plus elle donnerait un beau spectacle à la société ma Tante avait aussi mal de cœur. ${ }^{65}$

Mais la maladie qui semble omniprésente, voire obsédante dans les journaux des diaristes, y compris les plus jeunes, c'est la «maladie de poitrine », parfois nommée « hydropisie» : la tuberculose. De fait, plusieurs de ces jeunes Pétersbourgeoises y succomberont dans la fleur de la jeunesse. C'est notamment le cas de Natal'ja Šahovskaja qui précise, dans l'incipit de son troisième journal (1846, qu'elle n'achèvera pas puisqu'elle succombera l'année suivante à la maladie, à Rome), les circonstances malheureuses qui ont contraint sa famille à entreprendre ce voyage en Europe :

Samedi 25/13 juillet 1846. [...] Hélas c'est pour moi que ce voyage a été entrepris. Je tombai malade bien sérieusement le lundi 14 janvier 1846 [...] nous allâmes [au bal] Mathilde ${ }^{66}$ et moi déjà avec de la fièvre, je dansais beaucoup et me réveillais le jour suivant en crachant le sang, Mathilde aussi se réveilla malade et on nous mit mercredi toutes les deux au lit dans notre chambre à coucher à Petersbourg [...] Depuis mercredi je ne quittais plus l'étage d'en haut pendant plus de six semaines, au bout de ce temps je me sentis mieux [...] lorsque le 24 avril mercredi après diner je me mis à cracher le sang avec abondance et cela sans aucune raison. [...] Je continuais toujours à cracher le sang et j'allais si mal que tout à coup tous nos projets changèrent et Papa décida dans sa grande bonté pour moi que nous irions à l'étranger $[\ldots]^{67}$

Alors que la famille est de passage à Berlin, on fait venir un médecin pour examiner Natal'ja, sous le prétexte d'un... coup de soleil. Non seulement ce médecin n'est guère apprécié, mais ses conseils — vraisemblablement judicieux — ne seront pas suivis :

Papa voulut absolument consulter Schönlein, cette caricature, véritable vilaine poupée comme on en fait toujours pour les enfants - ces vieillards avec une

64. Natal’ja Šahovskaja, [Journal, 1841-1842], 1. 4v-7.

65. Gretchanaia, Viollet, éds., « Si tu lis jamais ce journal... », p. 266.

66. Marija (Matil’da) Mihajlovna Golynskaja, (1824-1848), cousine germaine de Natal’ja, diariste elle aussi, qui tient un journal parallèle à celui de Natal'ja durant ce voyage. Elle succombera également, un an plus tard, à la tuberculose.

67. Natal’ja Šahovskaja, [Journal, 1846], IRLI, f. 334, n 978, 1. 5-6. 
bouteille de vin à la main et une foule de verrues sur la figure et particulièrement sur le nez - voilà le vrai portrait de Schönlein et avec cela aucune aménité et bonhomie il n'est bon qu'à être pris en antipathie - aussi je n'ai pas tardé de nourrir ce sentiment pour lui. On l'appela pour ma figure et on ne le consulta que pour ma poitrine, il m'examina avec le stétoscope et m'envoya à Ems pour quatre semaines puis à Thoun et pour l'hiver à Florence ou Rome, on le consulta mais on ne fit rien, comme de raison, de ce qu'il dit. ${ }^{68}$

À Paris, la famille consulte à la fois un allopathe et un homéopathe. Le médecin de famille, un certain Zimmermann, qui soigne également en Italie Cléopâtre - tante paternelle de Natal'ja avec qui elle voyage et qui souffre elle aussi d'une «maladie de poitrine » — n'obtient guère plus de succès que le médecin berlinois :

Le court traitement de ma bien aimée Cléopâtre sera [bientôt] terminé, probablement en Russie elle ne suivra pas l'instruction que lui donnera Zimmermann et nous aurons la douleur de lui voir des souffrances terribles, quand je pense que six mois de plus passés ici aurait pus la guérir radicalement, [...] je ne sais que devenir de désespoir. Zimmermann dit que maintenant il serait très facile de la guérir dans six mois tandis que plus tard ce sera complètement impossible et que probablement elle aura le cancer, il la supplie de rester, [...] mais elle ne veut pas même en entendre parler je ne sais pourquoi, car je trouve que pour l'argent ce serait très faisable. ${ }^{69}$

Ces inquiétudes concernant Cléopâtre se retrouvent en écho dans le journal d'une autre cousine et contemporaine de Natal’ja, Praskov’ja Golynskaja :

J'ai éprouvé bien de la peine en voyant souffrir si fort ma bien-aimée Cléopâtre qui est indiposée ces jours-ci - Ah! Dieu veuille qu'elle souffre moins - si elle pouvait seulement se traiter ! mais comment ? et avec quoi ? - il est impossible à ce qu'on dit de passer un second hiver en Italie, ce qui lui aurait fait du bien pour sûr, car elle aurait entrepris une cure suivie - mais d'où prendre les moyens - et y consentirait-on en Russie ? Cartoni, Alertz, et Zimmermann disent que ce serait indispensable pr la santé de Cléopâtre et de Sophie, c'est même dangereux de rentrer en automne en Russie..$^{70}$

Quelques mois plus tard au cours du voyage en Italie, Matil'da Golynskaja, elle aussi atteinte de tuberculose, note dans son journal à propos de sa cousine :

Natalie se sentait tous les jours plus mal alors personne n'avait le cœur de sortir, ah ! Dieu veuille qu'elle guérisse. - mais je doute, M. *Uhland qui est avec nous dit qu'elle ne relèvera pas de cette maladie. - c'est affreux, si jeune et puis elle n'a pas envie de mourir. - Maintenant elle se sent beaucoup mieux, mais sa toux augmente toujours. - si les tubercules ne suppurent pas alors elle pourra peut être guérir, mais une fois la suppuration établit ce sera fini d'elle, - je ne puis m'imaginer comment la vie sera sans Natalie, je me suis tant habituée à

68. Ibid,1. 6-7.

69. Natal'ja Šahovskaja, [Journal, 1841-1842], op. cit., 1. 89-89v.

70. Praskov`ja Golynskaja, [Journal, 1841-1842], RGB, f. 336/II, carton 78, d. 2, 1. 19v. 
elle. - quel vide dans la maison. - j'espère que Dieu aura pitié d'elle et de toute sa famille c'est si triste d'aller au bal sans elle, et surtout de s'amuser en la sachant à la maison malade. ${ }^{71}$

Par ailleurs, les diaristes victimes de maladies manifestent constamment (du moins dans leur journal) une résistance d'ordre moral face aux épreuves physiques. Celleci peut soit prendre la forme de méfiance ou de moquerie vis-à-vis des médecins (c'est le cas du journal de Natal'ja Šahovskaja), soit se traduire par une grande fermeté en vue de combattre les faiblesses physiques et de préserver l'énergie vitale. Ainsi Ekaterina Vadkovskaja, âgée de cinquante-six ans, note-t-elle dans son journal de 1821 que, paralysée du côté droit (victime d'une hémiplégie), elle se force à écrire « la main gauche guidant la droite », et ce non pas afin d'en récupérer l'usage suivant les conseils médicaux, mais « uniquement» pour enregistrer ses « faits et gestes $»^{72}$.

Les aspects spécifiques de l'intimité féminine, telles que les menstrues ou la grossesse, sont également mentionnés, et ne semblent aucunement constituer des sujets tabous ${ }^{73}$. Elizaveta Šahovskaja inscrit dans son journal de 1825 que «d'un jour à l'autre [elle] attend le moment de [ses] couches », et se félicite du fait que son mari «l'accable de soins dans [son] état qui lui donne plusieurs incommodités ». Craignant d'être enceinte de jumeaux, Marija Bahmeteva relate cet échange avec sa belle-mère : «J'étois tous seul avec ma belle mère nous avons toujours parlé des couches et j'ai peur que je ne sois grosse et par malheur de deux comme tous les parens de mon mari ont eu $\gg^{74}$. La jeune Sof'ja Murav'eva explique que ses tantes l'empêchent de sortir pendant son «époque » (Journal, 1842) ; elle n'est d'ailleurs pas la seule à mentionner ces périodes d'indisposition - fait qui paraît tout à fait exceptionnel pour l'époque. Dans le journal de Varvara Ivanovna Lanskaja (née Odoevskaja ; 1790-1845), on trouve de nombreuses occurrences de cet ordre :

J'avais vu ma Cousine ; elle était malade d'une fausse-couche.

Le 28 Janvier 1810 ma cousine Lanskoï accoucha d'un Enfant mort.

Le 1 Janvier 1813 je me sentis mal et si mal, que je crus que j'allois accoucher.

Le 25 Sep. [1827] la première apparition de règles de ma fille Barbe. ${ }^{75}$

71. Maria (Matil’da) Golynskaja, [Journal, 1846-1847], RGB, f. 336/II, carton 78, d. 1, 1. 34.

72. Gretchanaia, Viollet, éds., « Si tu lis jamais ce journal... », p. 199.

73. Les menstrues ne sont mentionnées dans les journaux français que bien plus tardivement, dans la seconde moitié du Xx ${ }^{\mathrm{e}}$ siècle. Voir Françoise Simonet-Tenant, « L'Écriture de l'intimité féminine par les diaristes et les autobiographes », in Lila Ibrahim-Lamrous et Séveryne Muller, éds., L'Intimité, Clermont-Ferrand : Presses de l'Université Blaise Pascal, 2005, p. 101-115.

74. Journal depuis le 10 août 1805, par Marie de Bachmeteff, RGADA, f. 1256, op. 1, d. 1153, 1. 82 .

75. Varvara Ivanovna Lanskaja, [Journal 1806-1833], RGALI, f. 1337, op. 1, d. 129, 1. 1, 2, 5, $35 \mathrm{v}$. 
Quant aux notations qui se rapportent aux aspects corporels de la vie quotidienne, elles sont assez rares. Quelques diaristes décrivent leurs robes et tenues de bal (Marija Tolstaja), parfois pour s'en plaindre (Ekaterina Fredericks), critiquer celles d'autres femmes (Praskov'ja Golynskaja), ou admirent les costumes que porte la population des régions visitées ; on mentionne la venue, avant d'aller au bal, d'un coiffeur français ; mais dans l'ensemble, ces journaux féminins accordent étonnamment peu d'intérêt aux toilettes. On trouve également très peu de renseignements sur la nourriture ; si le rituel du thé semble être rigoureusement respecté au cours des voyages à l'étranger, ce sont les spécialités locales que relèvent les journaux : on déguste à Paris d'excellentes glaces de chez Tortoni, des pâtisseries nommées « plaisirs », et les figues d'Avignon paraissent « extraordinairement bonnes » ${ }^{76}$.

Le discours sur la nourriture, dans les journaux de Mme de Krüdener (17961800), répond aux exigences du code sentimental : elle ne mentionne que les nourritures simples, qui renvoient à la vie au sein de la nature : essentiellement les laitages comme la «crème » ou le « lait d'ânesse » ${ }^{77}$, et les fruits ${ }^{78}$. Elle s'indigne contre un «excellent » dessert, car ce luxe est à l'opposé de la «modération » et peut corrompre $«$ les mœurs ${ }^{79}$. Ce code continue à fonctionner plus tard: Marija Tolstaja mentionne régulièrement qu'elle dîne avec sa tante, mais n'entre jamais dans les détails du repas ; elle note cependant en 1821, en recourant à l'expression en russe, qu'elle a dîné seule « d'une excellente prostokvaša [lait caillé] ${ }^{80}$.

Quant à la mort, elle est souvent présente - qu'il s'agisse d'allusions à la sienne propre ou à celle de proches dont on se souvient ou que l'on commémore. Le discours sur sa propre mort peut être teinté d'humour comme, par exemple, dans le journal d'Ekaterina Vadkovskaja :

je vois passer un Corbillard qui bien vraiment avoit l'air de faire des visites, il s'est arreté a plusieurs portes, pour demander ou étoit le Mort qu'il venoit chercher, que n'a t'on pu à la mienne lui repondre que j'étois prête a l'occuper [...] J'ai envie de promettre ma pratique à cet homme, mais qu'il ne prenne pas la peine de venir s'infformer de mes nouvelles... ${ }^{81}$

Les réflexions sur la mort des proches ou d'autrui se développent dans un registre empreint de gravité, et aboutissent généralement à des conclusions philosophiques soit d'ordre général, soit en relation avec l'existence de la diariste. Sof'ja Murav'eva se souvient de la mort de sa mère en des termes particulièrement douloureux :

[...] je me rappelle très bien l'affreuse impression sans pareille que m'a causé sa mort, oh mon Dieu que je ne voudrais pas recommencer cet affreux moment, oh

76. Voir Gretchanaia, Viollet, éds., « Si tu lis jamais ce journal... », p. 275-297.

77. Mme de Krüdener, [Journal, 1799], GARF, f. 967, op. 1, d. 3, 1. 31v.

78. Mme de Krüdener, [Journal, 1799-1800], ibid., d. 3a, 1. 23v.

79. Gretchanaia, Viollet, éds., « Si tu lis jamais ce journal... », p. 153-154.

80. RGADA, f. 1280, op. 1, d. 134,1. 79.

81. Gretchanaia, Viollet, éds., « Si tu lis jamais ce journal... », p. 205. 
jusqu'à présent je ne peux pas me rappeler sans une peine bien vive, cet instant solennelle et horrible où j'étais debout et silencieuse, et comme tuée devant sa couche de souffrance ; non je n'oublierai jamais cet instant mais j'ai senti en moi tout à coup un changement prompt et moi qui deux jours avant ce jour à jamais gravé dans mon cœur, moi qui riait, qui était comme fille vive, turbulente, animée, d'une manière fatiguante, cruel ; eh bien depuis cette terrible perte je sens que je suis toute autre et que ma vivacité mon animation ont perdu la plus grande partie de leur turbulence ${ }^{82}$.

Le récit détaillé que rédige Kleopatra Mihajlovna Šahovskaja (dans un album réservé à cet usage) ${ }^{83}$ des derniers instants de ses nièces Natal'ja Šahovskaja et Matil'da Golynskaja ${ }^{84}$ - âgées toutes deux d'une vingtaine d'années - constitue un document rare et un témoignage émouvant. On y trouve de nombreuses précisions sur le discours religieux, les pratiques médicales et les usages liés à l'expression des dernières volontés.

\section{Voyages}

Le voyage comme événement important de la vie est un des thèmes prioritaires des journaux, qui occupe en entier ceux dits « de voyage $»^{85}$. Le plus ancien de ces journaux de voyage rédigés en français est celui de la baronne Natal'ja Mihajlovna Stroganova, intitulé « Pour mes sœurs. Sujet de critique à ceux qui l'aiment. C'est le titre du journal de mon voyage » (1780-1782). Natal' ja Petrovna Golicyna recopie sur un cahier à part des «Remarques sur mes voyages » (1783-1790). La comtesse Anna Ivanovna Tolstaja rédige une «Note de mon voyage l'an 1789 ». Elizaveta Vasil'evna Davydova nomme le sien, qui tient en cinq cahiers (1839-1844), non sans une pointe d'humour : « Journal voyageur et fantastique d'une jeune fille de 16 ans. Édition première et dernière ornée de beaux dessins de l'ouvrage de l'auteur ${ }^{86}$. On offre à la toute jeune Natal'ja Šahovskaja, à son arrivée à Paris, un cahier spécialement destiné à noter les impressions de son séjour à l'étranger ${ }^{87}$.

82. Journal. Mai 1842, RGB, f. 336/II, carton 78, d. 3, 1. 20v- 21.

83. Même s'il ne s'agit pas à proprement parler d'un journal, mais plutôt d'une chronique des décès au sein de la famille, de 1844 [?] à la fin du XIXe siècle, les entrées y sont datées. RGB, f. 336/II, carton 78, d. 19 .

84. Ibid., « Derniers instans de notre bien aimée Nathalie arrachées à notre tendresse le 10/22 Décembre 184 à Rome », 1.7-14v; «Derniers instans de notre bien aimée Mathilde arrachée aussi à notre tendresse onze mois après sa Cousine bien aimée »,1. 15-20.

85. Lorsque ce n'est pas le cas, le journal du quotidien - qui porte généralement le simple titre de « Journal » - peut se transformer à l'occasion en journal « de voyage »; ou l'inverse : si le support est le même, les frontières textuelles sont ténues, et dépendent de la situation de la diariste.

86. Voir les fragments des journaux de N.M. Stroganova, N.P. Golicyna, A.I. Tolstaja, E.V. Davydova in Gretchanaia, Viollet, éds., « Si tu lis jamais ce journal ».

87. Cahier portant la dédicace : « À ma bien chère Nathalie, Souvenir de Paris ». RGALI, f. 1337 , op. 1, d. 292. 
Si les jeunes filles de l'aristocratie sont encouragées à tenir un journal, le voyage - qui plus est à l'étranger - constitue une occasion toute particulière de recourir à cette pratique, la justifiant amplement aux yeux des proches comme de la diariste. Du fait que le voyage joue pour ces femmes un rôle important dans leur formation, son récit est destiné à conserver le souvenir des lieux visités comme des événements vécus, mais aussi à les partager avec les proches, et enfin à témoigner d'un degré élevé de culture.

Il n'est d'ailleurs pas rare que plusieurs personnes voyageant ensemble tiennent simultanément, «parallèlement », leur journal : c'est notamment le cas de Ekaterina Barjatinskaja et de sa fille Anna Tolstaja, de la princesse Elena Mihajlovna Golicyna (1776-1855) et de sa compagne, la comtesse Ekaterina Artem'evna Voroncova (1780-1836) ${ }^{88}$, ou encore de Natal'ja Šahovskaja et de trois de ses cousines germaines, Matil’da Golynskaja, Praskov’ja Golynskaja et Sof’ja Murav'eva $^{89}$.

Le voyage constitue une occasion spécifique, privilégiée, d'écrire, et le journal de voyage un « genre » à part entière. D'après les témoignages des diaristes ellesmêmes, la lecture, dans le cercle familial, de journaux de voyage est une pratique fréquente. De fait, les motivations de l'écriture viatique diffèrent quelque peu de celles qui gouvernent l'écriture diaristique. Il peut s'agir d'une commande :

Mon Pere exigea de moi, que j'écrivisse jour par jour, ce que je veroit, et surtout ce que j'eprouverois, en voyant les Chef-d'CEuvres de l'Art, et Ceux de la Nature ; J'ai Obeis $[\ldots]^{90}$

précise Ekaterina Vadkovskaja lorsqu'elle recopie pour sa fille ce «Journal du voyage de Mademoiselle la Comtesse de Czernichew à la suite de Sa Majesté l'Impératrice Catherine II : L'année 1787 », qu'elle avait rédigé à cette occasion. La plupart des journaux commencent in medias res, soit au moment du départ de Russie, soit au moment de l'arrivée à destination :

Ce 20/8 Août 1841. Paris Hôtel du Helder. Me voilà une seconde fois aux pays étrangers, j'en suis contente et fâchée en même temps, contente car je désirais revenir à Paris et aller en Italie et fâchée parce que je m'amusais au-delà de l'expression cet été étant en famille $[\ldots]^{91}$,

tel est l'incipit du deuxième journal de voyage de Natal’ja Šahovskaja. Plus loin, elle précise les raisons de cette pratique, et ses limites :

Via Gregoriana $N^{\circ} 42$. Rome, 18 mai 1842 . Mercredi. Il y a plus de sept mois que j'ai discontinué d'écrire mon journal, mais maintenant me trouvant dans une

88. [Journaux, 1826-1835], RGB, f. 64, carton 111, d. 1-6.

89. Voir note 14 de l'introduction « À la rencontre des journaux personnels ».

90. Gretchanaia, Viollet, éds., « Si tu lis jamais ce journal... », p. 210.

91. Natal’ja Šahovskaja, [Journal, 1841-1842], 1. 2. 
ville si célèbre en souvenir je veux me forcer d'écrire de nouveau tout ce que je verrai car après lorsque je serai en Russie j'aimerai à rouvrir ce livre qui me rappellera plus que tout autre chose le temps que j'ai passé aux pays étrangers, ce temps que j'en suis sûre je regretterai toujours, et puis pour mon instruction je trouve que je dois inscrire tout ce que je verrai sans quoi je l'oublierai ; il m'aurait été aussi très agréable de décrire dans ce livre les différentes impressions par lesquelles j'ai passé et qui ont été plus nombreuses que jamais, mais ceci est complètement impossible car il y a une quantité d'impressions que je n'aurais pas voulu qu'on connaisse (et probablement à notre retour en Russie plusieurs personnes voudront lire ce journal) $[\ldots]$, donc je suis décidée à ne parler ici que de ce que je vois et à ne décrire que bien peu d'impressions. ${ }^{92}$

Mais c'est l'incipit de son troisième (et dernier) journal conservé (1846) qui lui fournit le prétexte d'une réflexion sur la différence entre écriture diaristique proprement dite et écriture viatique : les distinguant soigneusement l'une de l'autre, elle en théorise en quelque sorte la différence ${ }^{93}$ :

Baden-Baden - Maison Kleinmann, Samedi 25/13 juillet 1846.

Me voici donc une troisième fois à l'étranger ! serait-il possible que cette fois je n'écrive pas mon journal, moi qui m'en faisait toujours le martyre à mes deux premiers voyages ; [...] la pensée m'est venue, ce matin, que peut-être plus tard je regretterai de n'avoir pas fait le journal de mon troisième voyage, certainement que je ne me tourmenterai plus comme aux deux premiers, mais j'écrirai quand j'en aurai la disposition et je pense qu'elle me viendra souvent car je voudrais conserver un souvenir écrit de ce voyage, en le relisant plus tard après plusieurs années les impressions passées s'éveillent et reviennent toutes vives, tandis qu'elles s'engourdissent et disparaissent complètement si rien de matériel ne les fait revivre et c'est dommage, car les souvenirs et impressions de voyage sont agréables, [...], c'est tout autre chose que ce qu'on écrit dans une situation stable, le journal d'un voyage n'est pas aussi intime que celui qu'on écrit dans une vie sédentaire dans la patrie là où on n'a affaire qu'avec ses propres sentiments et pensées qui vous viennent d'une vie intérieure. Le journal d'un voyage est tout extérieur, car on ne décrit que les impressions que la vue des objets extérieurs font rejaillir sur vous..$^{94}$

Sur le plan de la présentation matérielle, de l'organisation graphique et spatiale, la plupart des journaux de voyage présentent d'ailleurs des traits caractéristiques qui les différencient des journaux ordinaires : sont systématiquement mis en valeur, dans le fil du texte, des repères tels que lieux, événements, nom des personnes rencontrées - soit en les inscrivant en marge, soit en les soulignant.

De quels voyages s'agit-il ? Certains journaux relatent des voyages en Russie, d'autres dans les villes d'eaux (Baden, Spa...), mais pour la plupart, il s'agit de

92. Ibid., 1. 34-35.

93. Ses remarques pertinentes ne renvoient-elles pas à la distinction toute récente entre journal intime et journal extime, tel qu'il est pratiqué par des écrivains comme Michel Tournier ou Annie Ernaux ?

94. IRLI, f. 334, d. 978, 1. 1. 
voyages au long cours dans différents pays d'Europe de l'Ouest qui généralement convergent vers l'Italie, but final. On quitte la Russie soit par voie terrestre, soit par bateau. Ainsi partant d'Odessa, Elizaveta Vasil'eva voyage en bateau jusqu'à Venise, en passant par Constantinople, Smyrne, Athènes, Trieste. Durant le séjour en Italie, qui dure au moins six mois ${ }^{95}$, elle visite Venise, Padoue, Vérone, Milan, Pavie, Gênes (un mois), Livourne, Sienne, Florence (deux mois), puis séjourne à Rome. Si l'on croise les journaux discontinus de Natal'ja Šahovskaja et de sa cousine, Praskov'ja Golynskaja (qui commencent à Marseille), on obtient l'itinéraire presque complet du deuxième voyage (d'environ dix-huit mois) qu'effectue cette famille (1841-1842) : de Moscou à Saint-Pétersbourg, de là en bateau (sur l'Amsterdam) jusqu'à Copenhague, puis Le Havre, Rouen et Paris, où la famille séjourne environ deux mois. On se dirige ensuite vers Gréoux (en Haute-Provence, pour une cure de trois semaines) en passant par Lyon, Avignon, Aix, Marseille et sa région (une semaine). Puis vient l'Italie, partie essentielle du voyage: Gênes, Livourne, Pise (séjour de sept mois), Rome (un mois et demi), Naples et ses environs (un mois), Florence, Bologne, Ferrare, Venise, et retour à Saint-Pétersbourg par Florence, l'Allemagne et la Pologne.

Ces voyages se déroulent généralement en groupe familial élargi. Elizaveta Vasil’eva précise que cinq personnes y participent : «Maman, la Comtesse [amie de sa mère], Melle Mam [gouvernante anglaise ?], Linchen [probablement sa petite sœur, ou bien la fille de la comtesse] ». Elizaveta Davydova voyage avec son oncle et sa tante, leurs enfants et des domestiques ; au cours de leurs différents voyages, les Šahovskie rassemblent plusieurs tantes et nièces (cousines entre elles), deux générations donc, assorties d'un ou deux domestiques venus de Russie et d'autres recrutés sur place ${ }^{96}$.

La description des moyens de transport occupe une place de choix dans les journaux : bateaux à vapeur ou pyroscaphes (l'Amsterdam, l'Aigle, le Pharamond, le Lombardo, le Léopold II...), dont on décrit par le menu - entre tempêtes et incendies - l'aménagement intérieur, l'amabilité du commandant et du personnel navigant, ainsi que la « société » à bord, plus ou moins fréquentable ; les différents moyens de transport terrestre (calèches, coupés, diligences, voiturins, voitures de poste), et le problème bien connu du changement de chevaux aux relais de poste. Mais la véritable nouveauté est le chemin de fer (en France et en Allemagne), dont on apprécie avant tout la rapidité :

Quelle différence entre le chemin de fer de Paris et celui de Petersbourg, comme on va plus vite sur celui de Paris, on vole mais aussi il y a souvent des accidents

95. RGALI, f. 1337, op. 1, n 24. Commencé le 25 Juillet 1836, le journal s'interrompt le 12/ 24 mars 1837, alors que l'auteur se trouve toujours à Rome ; nous ignorons donc quand s'achève ce voyage.

96. Le père de Natal’ ja, Valentin Šahovskoj, reste à Paris avec sa seconde épouse, enceinte, et ce groupe de femmes voyage en Italie sans accompagnateur masculin, ce dont elles se plaignent: "C'est si désagréable de voyager sans homme, aussi lorsqu'on en trouve de complaisant, on en est enchanté », affirme sa fille (Natal'ja Šahovskaja, [Journal, 1841-1842], 1. $39 \mathrm{v})$. 
malheureux. Nous avons été trois fois sur le chemin de fer à Paris deux fois pour aller à Versailles et la troisième fois pour aller à St Germain [...], on passe par des cavernes, des souterrains, c'est tout à fait amusant..$^{97}$

Amusants sont, dans le train, les réflexes des diaristes, identiques à ceux du voyage en bateau : on décrit, examine et évalue, en termes de «fréquentables » ou non, les passagers qui partagent le compartiment.

À côté de la date de chaque entrée, les diaristes notent généralement le lieu exact de leur villégiature : soit les hôtels (on pourrait établir, à partir de leurs commentaires, un véritable guide étoilé des hôtels de l'époque), soit les logements loués pour des périodes plus longues. Ainsi, à Strasbourg, on revient dans le même hôtel à chaque passage, l'hôtel de France ; à Toulon, c'est l'hôtel du Cheval-Noir ; à Gênes, l'hôtel de France se révèle « parfaitement détestable », l'hôtel du Globe et celui de l'Aigle-Noir, à Livourne, « passablement mauvais » (les chambres y sont humides, sombres et froides), tandis que le Grand-hôtel Saint-Marc offre « quatre bonnes chambres au soleil et fort jolies »; à Florence, l'hôtel du Nord déplaît, mais on est «fort bien logées» à l'hôtel du Pélican, et «servies parfaitement». À Venise, faute de choix, on s'installe à l'hôtel Royal-Danielli :

Nous nous promenâmes plus d'une heure pr chercher des appartemens mais les hôtels de l'Europe, du Lion Blanc et de l'Angleterre, ainsi que de l'étoile d'Or étaient tous pris - ns nous décidâmes donc pr cet hôtel-ci nous n'avons que trois petites chambres, ms ns en espérons avoir davantage après le départ de 1'Archiduc Frédéric qui loge à côté de nous, ce qui m'amuse beaucoup. ${ }^{98}$

À Rome, le domestique russe d'une de leurs connaissances recommande « une maison dont l'hôte est russe » : les chambres y sont « assez jolies, mais sombres et sales ». À Naples, elles trouvent une « maison très commode, tout à fait charmante, sur une place magnifique ». Lorsqu'on s'installe pour un certain temps dans un logement loué, on engage des domestiques ( « À Gênes, notre service se compose de trois domestiques »), on s'empresse de louer un piano. Mais l'inconfort fréquent est souvent la cause de refroidissements ; on dort souvent « à deux sur le même lit », et il arrive qu' on passe « la nuit sur la table, les lits étant pleins d'insectes ${ }^{99}$. Enfin, la question d'argent n'est pas étrangère à ces choix :

[Rome. Mardi 17/5 Mai 1842] pendant ce temps arriva une lettre de Papa qui nous envoyait une lettre de change, ceci nous fit un plaisir vraiment très grand, car nous n'avions plus d'argent et ne savions que faire. ${ }^{100}$

97. Natal’ja Šahovskaja, [Journal, 1841-1842], op. cit., 1. 11v. La ligne Paris-Versailles a été inaugurée en 1840, celle de Paris à Saint-Germain-en-Laye en 1837. Ces mêmes diaristes prennent aussi le train, en 1842, entre Strasbourg et Bâle (inauguré en 1840).

98. Praskov’ja Golynskaja, [Journal, 1841-1842], RGB, f. 336/II, carton 78, d. 2, 1. 44.

99. Ces précisions sont extraites de différents journaux de la famille Šahovskoj (Natal’́ja Šahovskaja, [Journal, 1841-1842] ; Praskov’ja Golynskaja, [Journal, 1841-1842]).

100. Natal`ja Šahovskaja, [Journal, 1841-1842], 1. 40. 
Lors des séjours prolongés dans les villes étrangères, les occupations ne se réduisent pas aux visites et promenades touristiques : on prend des leçons de langue et de musique, on lit dans la langue du pays, on va régulièrement aux spectacles et aux bals. On fréquente de nombreuses connaissances ou encore la parentèle de Russie, également venue en villégiature, et les lettres de recommandation facilitent démarches et contacts avec les autochtones et un milieu largement cosmopolite; mais les rencontres de voisinage sont également appréciées.

\section{Formes de sociabilité}

Les journaux constituent un lieu privilégié pour observer l'ensemble des relations interpersonnelles - qu'il s'agisse du jeu des relations sociales, de la constellation familiale, ou encore celles au sein du couple : ils offrent en effet un point de vue « de l'intérieur », différent de celui que peut refléter par exemple la correspondance adressée à un tiers. Ce point de vue, certes éminemment subjectif, reste proche du vécu au jour le jour. Au-delà de la subjectivité du témoignage ${ }^{101}$, l'abondance des journaux permet de dessiner les contours d'un mode de vie partagé par la plupart des diaristes, mais aussi de souligner les particularités du vécu de chacune d'elles.

\section{Relations sociales}

L'aspect le plus frappant de ces journaux est l'abondance de noms propres : ils constituent un véritable réseau relationnel, puisqu'on retrouve souvent cités, d'un journal à l'autre, les mêmes noms de famille. Il s'agit des familles de l'aristocratie, toutes plus ou moins célèbres, auxquelles appartiennent les diaristes. La fréquence de ces occurrences témoigne de l'homogénéité et de la densité relationnelle au sein de cette classe sociale, du moins telle qu'elle est représentée dans ces écrits. On se rend mutuellement visite, se rencontre aux bals, aux spectacles, en promenade, aux parties de plaisir, aux eaux et aussi à l'étranger, où l'on retrouve ses connaissances de Moscou ou de Pétersbourg.

C'est surtout au cours des voyages dans les pays étrangers qu'ont lieu les rencontres avec des personnes d'autres milieux. Dans les moyens de transport collectifs (bateau, train), la qualité de l'entourage est évaluée en fonction du comportement et du degré d'éducation - d'après des critères tels que la manière de parler français et de se comporter auprès des dames.

101. Philippe Lejeune souligne l'incompatibilité de l'écriture diaristique (écriture du présent) et de la fiction et, différenciant soigneusement les notions de subjectivité et de fiction, définit le journal, tout subjectif soit-il, comme «antifiction». « Le journal comme "antifiction"», Poétique, 149, fév. 2007, p. 3-14. 
Ces séjours à l'étranger offrent constamment l'occasion de lier de nouvelles connaissances, et d'élargir le cercle des relations. Voici comment est décrite l'une de ces rencontres, lors d'un séjour prolongé à Pise de la famille des Šahovskie ${ }^{102}$ :

Lilia alla seule au troisième bal de la cour, les Paira [des voisines] y étaient aussi et elles tachèrent de faire connaissance, Sophie parla la première à Lilia [...]. C'est de cette manière qu'Emilie apprit qu'elle était près de Lilia, après ce bal elles nous engagèrent à plusieurs soirées, on allait de chez nous presque deux fois la semaine, nous fîmes connaissance chez eux avec plusieurs personnes, premièrement avec Fanny de Champigny [...]. Chez les Paira ns fîmes encore connaissance avec Monsieur et Madame *Senden, qui m'avait l'air d'un couple si heureux, puis avec plusieurs Messieurs très peu interessants [...]. Vers la fin de nôtre séjour nous recevions les jeudi soir et nous nous amusions beaucoup.

En fait de russe nous ne connûmes [à Pise] que Madame *Linden, Monsieur Meindorff, qui vint chez nous en passant par Pise et Madame *Tatischoff, qui etait avec sa sœur et ses enfants, c'est une famille fort peu interessante et que nous connûmes fort peu. ${ }^{103}$

À Livourne, la baronne d'Oechsen leur rend visite tandis que de leur côté, elles rendent, toutes ensemble, visite au Consul de Russie ; à Gênes, elles fréquentent la princesse Butera.

Le récit d'une autre diariste contemporaine, Praskov'ja Golynskaja, indique que ces jeunes filles pouvaient parfaitement se permettre de nouer des connaissances en dehors de l'étiquette, du moins en Italie :

Nous avons continué à nous baigner tous les jours [...], et c'est dans l'eau que nous fîmmes connaissances avec les filles de notre hôte le Prince Strongoli, l'une se nomme Camille [...], et l'autre Julie.104

Les rencontres de hasard ne sont pas non plus exclues ; certains journaux témoignent du fait - assez surprenant - que des jeunes filles (Natal'ja Šahovskaja et l'une de ses cousines) ont l'occasion, en visitant Capri, de passer un long moment, sans chaperon, avec deux jeunes hommes (dont l'un est musicien, l'autre peintre).

\section{Relations familiales}

Quel que soit l'âge des diaristes, la vie familiale occupe une place centrale dans les journaux - qu'il s'agisse de la relation au sein du couple, avec les enfants, ou avec la parentèle élargie.

L'un des thèmes principaux est la séparation d'avec les personnes chères. Dans les tout premiers journaux, il prédomine parmi d'autres, liés aux relations

102. Au cours de ce voyage, la compagnie est composée exclusivement de femmes (tantes et cousines de Natal'ja).

103. Natal’ja Šahovskaja, [Journal, 1841-1842], 1. 32-33v.

104. Golynskaja, Praskov`a Mikhajlovna, Journal, 1841-1842, 1. 20. 
affectives. Il est présent dans les journaux de voyage de Natal'ja Stroganova qui a quitté « ses sœurs pour la première fois de [sa] vie » ${ }^{105}$, et de Ekaterina Barjatinskaja, « séparée, peut-être pour toujours, de [sa] chère fille »106, ainsi que dans les journaux de la vie quotidienne de Natal'ja Golicyna qui éprouve à Moscou « un ennui et un chagrin terrible » après le départ de son mari ${ }^{107}$ et, plus tard, « une peine infinie » à se séparer d'avec ses fils partis faire leurs études à Strasbourg ${ }^{108}$. Il en va de même pour Ekaterina Kvašnina-Samarina qui, partie pour l'été à la campagne, « se transporte en imagination » auprès de ses amies lointaines ${ }^{109}$. Par conséquent, la réception des lettres provenant des êtres aimés et les nouvelles les concernant constituent un événement important, dûment enregistré dans le journal. La description des retrouvailles traduit une joie extrême. Retrouvant ses fils en France, Natal' ja Golicyna note dans son journal de voyage : « [...] une séparation d'un ans et pres de 4 mois pour une mere tendre comme je la suis a pu bien causé un santiment qu'il me seroit impossible d'exprimer ${ }^{110}$.

Dans le Journal épistolaire qu'elle adresse à ses sœurs «éloignées de 700 verstes » durant l'année 1826, la princesse Elizaveta Mihajlovna Šahovskaja (1797-1877) ${ }^{111}$ ne cesse d'assurer ces dernières, à chacune des entrées, de son intense affection :

Ah ! si tu savais le désir ardent que j'éprouve souvent de te revoir, de me retrouver au milieu de vous, de vous serrer chacune dans mes bras, si tu savais tout ce qu'il me faut de peine pour le calmer, et comme j'y réussis rarement !112

Marija Bahmeteva et Elizaveta Šahovskaja, qui soupirent après leur bien-aimé momentanément absent, enrichissent ce thème de la séparation par la description des tourments amoureux, l'expression des doutes et de toutes sortes d'inquiétudes ${ }^{113}$.

Le journal devient progressivement un lieu de réflexions sur diverses relations avec le bien-aimé ou l'époux, les enfants, les parents, les amis, l'entourage - et de mise au point des sentiments. Ces réflexions peuvent s'adresser directement à la personne qui en est l'objet, et le journal revêt alors la forme de la conversation ou de la lettre. C'est ainsi qu'Elizaveta Šahovskaja «parle» dans son journal avec son fiancé, qu'elle épouse quelque temps plus tard, l'assurant de son amour, lui faisant

105. Gretchanaia, Viollet, éds., « Si tu lis jamais ce journal... », p. 72.

106. Ibid., p. 105.

107. Remarques sur des événements de ma vie (1781 ?-1783), RGB,f. 64, carton 117, d. 1,1. 7.

108. Ibid., 1. 39v.

109. Gretchanaia, Viollet, éds., « Si tu lis jamais ce journal... », p. 133.

110. Remarques sur mes voyages (1783-1790), RGB, f. 64, carton 113, d. 1,1. 31.

111. Il s'agit de la sœur de Valentin Šahovskoj, futur époux d'Elizaveta Muhanova.

112. [Journal, 1826], GARF, f. 1738, op. 1, d. 26,1. 1.

113. Voir Gretchanaia, Viollet, éds., « Si tu lis jamais ce journal... », p. 188-189. 
des reproches, puis se repentant de sa «vivacité »114. Elle affirme à son bien-aimé qu'elle «ne prévoit ou plutôt ne cherche dans [leur] mariage que [leur] bonheur commun », se propose de lui apporter en guise de dot « un cœur qui t'adore, et le désir ardent de faire ton bonheur », et prévoit de se soumettre à la volonté de son futur époux : «La perfection du mariage consiste dans la réunion de deux volontés - Je dois donc faire mon étude principale à me conformer à la tienne $»^{115}$. Leur fille Natal'ja s'extasie devant les couples qui donnent une impression d'harmonie, car « il est si agréable de voir qu'un mari et qu'une femme s'aiment», et affirme que «le véritable bonheur ne peut exister qu'à la maison avec les siens $»^{116}$.

Natal'ja Fedorovna Bodé (née Kolyčeva ; 1790-1860), dans son journal destiné à être lu après sa mort par son mari, affirme qu'elle met « tout son bonheur à ne pas quitter une minute » celui qu'elle « aime à la folie » et dont « les aimables caresses la rendent si heureuse », et qu'elle doit également ses « heures de jouissance » à ses enfants, ces «petits anges qui font le bonheur et quelque fois le tourment de ma vie $»^{117}$.

Les femmes diaristes se sentent responsables de l'atmosphère familiale et considèrent de leur devoir de maintenir des relations harmonieuses, dont l'absence est perçue comme une épreuve personnelle. Le journal devient alors le dépositaire des plaintes, résolutions et espoirs des diaristes. S'apprêtant à revoir sa sœur de retour de l'étranger, au caractère réputé difficile, Olga Orlova-Davydova écrit : « $\mathrm{O}$ ! Mon Dieu, aide-moi d'être bien, bien douce et bonne avec elle, je l'écris pour me rappeller que j'en prends la résolution ${ }^{118}$.

Natal'ja Golicyna fait état dans son journal des « terribles disputes » entre elle et sa sœur par suite du partage de l'héritage de leur mère défunte ${ }^{119}$. Elle tire de ce conflit une leçon morale pour ses enfants :

J'ai detaillé tous ce malheureux partage et tous les desagréman que cela m'a occasionné, pour servir de leçon à mes enfans et pour qu'ils sache qu'une tel conduitte mercenaire est non seuleman prejudissiable, mais ne porte aucun profie, et la tache reste ineffassable pour la vie..$^{120}$

Mme de Krüdener consacre plusieurs pages de ses journaux à la description de ses relations, qui ne sont pas exemptes de travers, avec son mari, sa fille, son fils et sa belle-fille. Elle se montre également préoccupée par la manière, souvent brusque, dont elle traite sa femme de chambre, tout comme Elizaveta Šahovskaja qui, se

114. Gretchanaia, Viollet, éds., « Si tu lis jamais ce journal... », p. 225-236 ; Elizaveta Šahovskaja, Journal commencé six semaines après mon mariage (1825), GARF, f. 1738, op. 1, d. 8.

115. Elizaveta Šahovskaja, [Journal, 1824], RGB, f. 336/2, carton 47, d. 4.

116. Natal’ja Šahovskaja, [Journal, 1841-1842].

117. Gretchanaia, Viollet, éds., « Si tu lis jamais ce journal... », p. 188-189.

118. Ce qui s'est passé de plus remarquable pour moi depuis notre retour de Kamenka, 1. 6.

119. Remarques sur des événements de ma vie, $1.30 \mathrm{v}-32 \mathrm{v}$.

120. Ibid., 1. 33v. 
reprochant son irritation vis-à-vis des domestiques, s'efforce de se justifier tout en prenant la décision de se corriger ${ }^{121}$.

Les mères décrivent en détail leur relation avec leurs filles. Mme de Krüdener fait, en 1800, l'éloge de la sienne et, insistant sur le climat amical et chaleureux qui règne entre elles, souligne les avantages de l'éducation qu'elle, sa mère, lui a procurée ${ }^{122}$, mais s'accuse parfois de l' «injustice» de ses reproches « envers un ange de docilité »123. Ekaterina Vadkovskaja adresse son journal des années 1820 à sa fille aînée qu'elle affectionne à la manière de Mme de Sévigné, tandis que ses relations avec sa fille cadette semblent très tendues ${ }^{124}$. Elizaveta Šahovskaja décrit en 1834 le comportement de sa fille Natal'ja, âgée de huit ans :

On peut avec elle avoir quelquefois des conversations si interessantes, elle a une grande intelligence - elle est capable d'aimer fortement, mais exclusivement elle me donne la première place dans son Cœur. ${ }^{125}$

À leur tour, les filles rendent compte de différents sentiments qui naissent de leurs rapports avec leur mère et parfois belle-mère. Olga Orlova-Davydova se sent jalouse d'une de ses sœurs que, croit-elle, sa mère lui préfère ${ }^{126}$. Aleksandra Grigor'evna Černyševa (épouse Murav'eva ; 1800-1832) consacre son journal de 1816 à la description de ses torts qui la privent de la tendresse de sa mère, torts qu'elle juge avec virulence en promettant de corriger son attitude :

Je suis emportée c'est un malheur, mais je me corrigerai parce que je veux me corriger et parce que si je dis je le veux je le pourrai c'est une grande difficulté mais je surmontrai cette difficulté et plus ce sera difficile et plus j'aurais de plaisir a me surmonter, suis je donc un animal ou une piere pour ne pas me corriger est ce que je n'aime pas maman assez pour tout sacrifier pour elle vie même s'ille je sens qu'il me couterai moins de lui sacrifier ma vie, que ma colere dans un moment d'emportement et c'est pourquoi je mets mon ambition et mon honneur à me corriger, je renderais maman heureuse un jour, si elle ne m'aime pas apresent je ne le merite pas, et je la forcerai de m'aimer un jour par ma conduite $[\ldots]$ je ne peux pas supporter l'idée que maman me prefere mes sœurs, mais quelle amour propre de ne vouloir pas que maman aime mieux Sophie que moi. ${ }^{127}$

Sof'ja Murav'eva est, elle aussi, tourmentée par le remords d'une mauvaise conduite. Elle se reproche «la faute énorme» (sans doute un mensonge) qu'elle

121. Journal commencé six semaines après mon mariage.

122. Gretchanaia, Viollet, éds., « Si tu lis jamais ce journal... », p. 155-163.

123. Ibid., p. 162.

124. Ibid., p. 199-208.

125. RGB, f. 336/2, carton 47, d. 7,1. 16v.

126. Ce qui s'est passé de plus remarquable pour moi depuis notre retour de Kamenka.

127. GARF, f. 1153 , op. 1, d. $123,1.3-4$ v. 
doit cacher et qui l'empêche d'être franche avec tous ses parents ${ }^{128}$. L' " examen de conscience » qu'elle entreprend dans son journal a pour but de l'aider à sortir de « cet affreux labyrinthe de mensonges et de dissimulations », et de rendre ainsi « un peu de joie » à son père et à sa belle-mère qu'elle affectionne.

De manière générale, le journal intime sert à expliciter différents problèmes de communication, principalement dans le domaine affectif, à proposer sa propre vision de ces problèmes et à réfléchir sur les moyens de les résoudre.

La nouvelle génération de diaristes, jeunes filles nées autour des années 1820 , semble nourrir moins d'illusions quant à la possibilité de relations harmonieuses avec leur entourage. Elles n'hésitent pas à parler du peu de sympathie que leur inspirent certains parents, tout en maintenant l'image idéalisée des autres. La fille d'Elizaveta Šahovskaja, Natal'ja, et ses jeunes cousines voyageant en Europe en compagnie de leurs tantes n'espèrent pas adoucir les tensions à l'intérieur de ce groupe familial. Elles préfèrent une tante, Cléopâtre, à toutes les autres, et se moquent fréquemment d'une autre de leurs tantes, Catherine. Leurs journaux commentent la situation difficile dans laquelle se trouve Natal' ja, après le second mariage de son père, face à sa belle-mère. L'une de ses cousines écrit à propos de l'arrivée du père de Natal'ja et de sa nouvelle épouse à Naples en 1846 :

Cela me fit peine à voir Natalie si souffrante de leur arrivée. Pendant plusieurs jours elle était tout à fait abattue. Pauvre amie. Comme elle doit souffrir d'avoir une belle-mère. La position de belle-fille doit être affreuse. ${ }^{129}$

\section{Relations au sein du couple}

Presque tous les journaux ${ }^{130}$ témoignent du fait que le mariage résulte du libre choix des conjoints - un choix qui n'est pas toujours dépourvu d'obstacles.

Elizaveta Šahovskaja relate les réticences de la famille de son futur époux vis-àvis de son mariage - officiellement, en raison de l'âge du jeune homme, que l'on éloigne alors pour de longs mois; plus probablement, du fait qu'Elizaveta ne possède guère de biens à apporter dans la corbeille... ${ }^{131}$. Plus tard, elle se plaindra du fait que son mari lui cache ses activités de franc-maçon.

128. Gretchanaia, Viollet, éds., « Si tu lis jamais ce journal... », p. 301.

129. Marija (Matil’da) Mihajlovna Golynskaja, [Journal, 1846-1847], 1. 60v-61.

130. Exceptés deux journaux de la fin du XVIII ${ }^{\mathrm{e}}$ siècle, où il semble s'agir de mariages arrangés ; ces deux mariages ont été bénis par l'impératrice Catherine II. Natal’ja Golicyna : «je fu promise le $\underline{15}$ de Janvier de lan 1766 au Prince Владимир Борисович Голицын [Vladimir Borisovič Golicyn] qui étoit colonel dans le Regiment de Пермской пехотной [d'infanterie de Perm] », in Gretchanaia, Viollet, éds., « Si tu lis jamais ce journal... », p. 82. Et Ekaterina Vadkovskaja : «Le 2. de Septembre 1821. Il y a aujourd'hui 33 ans que j'ai été Marié à la Chapelle du Palais d'hiver à St Petersbourg », Ibid., p. 199.

131. Elizaveta Šahovskaja, Journal depuis le 22 Mai 1822, RGB, f. 336/2, carton 47, d. 2. 
Au cours d'un voyage en Italie, les nièces de Kleopatra (la belle-sœur d'Elizaveta) Šahovskaja souhaitent fébrilement lui voir épouser un Italien « bien sous tous rapports », qui lui fait la cour : «Que n'est-il en mon pouvoir de lui trouver un être aussi parfait qu'elle qui puisse la comprendre, l'apprécier - s'unir à elle pour la rendre heureuse ${ }^{132}$.

Quelques journaux expriment, au long des pages, les vifs sentiments des jeunes filles à l'égard de l'élu de leur cœur. Une fois le mariage célébré, le ton change parfois : soit le journal s'interrompt définitivement, soit il fait état de plaintes, de déceptions ou de craintes quant à la fidélité de l'élu. Natal'ja Bodé ne tient « [son] journal, ou plutôt [ses] pensées et réflexions »133, que pour enregistrer les « démêlés » avec son mari et ses peines. S'adressant à lui, elle se plaint de sa « froideur et indifférence ». Après son mariage, Marija Bahmeteva transforme son journal en un monologue fermé, un soliloque douloureux. Convaincue que son mari ne l'aime plus, elle décrit «l'enfer » qui règne dans son âme ${ }^{134}$.

L'aspiration à la sérénité et à l'équilibre affectif marque plusieurs journaux. Olga Orlova-Davydova écrit dans son journal de 1843 à propos de son mari : «Je ne puis m'habituer à ne pas être comprise de Vladimir et c'est ce qui me met du trouble dans l'âme »135. Dans les comptes rendus fréquents des inconvénients du ménage, les femmes diaristes affirment généralement leur bonne volonté, justifient leur conduite par un amour excessif et une sensibilité extrême, sans cesser pour autant de constater le manque de bonne entente avec leur époux.

\section{Autres relations}

En dehors des relations familiales, ce sont l'amitié et les inclinations amoureuses qui tiennent une place privilégiée dans les journaux des jeunes filles dès la fin $\mathrm{du}$ XVIII siècle. Elles ont besoin d'amies et se réjouissent d'en avoir. L'amitié amoureuse, qui s'appuie sur les modèles littéraires de l'époque, lie parfois les diaristes à d'autres femmes ${ }^{136}$. Quant à l'amour, il n'apparaît dans le journal de jeune fille qu'une fois devenu « légitime », c'est-à-dire avec la perspective de mener prochainement au mariage. Ce n'est qu'à cette condition que les diaristes, comme Marija Bahmeteva, Varvara Suhotina ${ }^{137}$ ou Elizaveta Šahovskaja, parlent dans leurs journaux de l'amour qu'elles portent à l'homme qui deviendra effectivement leur époux.

132. Praskov’ja Golynskaja, [Journal, 1841-1842],1. 15.

133. Gretchanaia, Viollet, éds., « Si tu lis jamais ce journal... », p. 188.

134. RGADA, f. 1256, op. 1, d. 1153,1. 66.

135. RGB, f. 219, carton 92, d. 10,1. 14.

136. Voir Elena Gretchanaia, Catherine Viollet, «Ressusciter ces voix oubliées. Journaux féminins russes en langue française (1780-1854) », in Gretchanaia, Viollet, éds., «Si tu lis jamais ce journal... », p. 53-55.

137. [Journal, 1811], RGADA, f. 1280, op. 1, d. 360. 
Les inclinations amoureuses sont enregistrées sous la forme de petites scènes où entrent en jeu les «chevaliers galants » ou personnages masculins intéressant les diaristes. Sont alors reproduites, dans les journaux de Marija Tolstaja, de Natal’ja Šahovskaja, de Praskov`ja Golynskaja, de Ekaterina Fredericks ${ }^{138}$, les conversations lors des bals et des rencontres dans les salons, les gestes et les regards, les mots d'esprit échangés avec les cavaliers. C'est tout un jeu de séduction, qui paraît souvent quelque peu désinvolte et piquant, d'une grande liberté de ton, d'après les dialogues (et jugements) que rendent les journaux. Le jeu reste visiblement innocent, ne sortant pas des limites que posent les convenances, et le sentiment semble se trouver toujours maîtrisé par un raisonnement plein de sagesse.

Les diaristes reconstruisent et analysent ainsi le réseau de leurs relations, leur univers affectif, qui revêt une importance cruciale. En traçant les configurations affectives, le journal permet aux diaristes de les saisir avec plus de netteté, de les régler, voire même de les redessiner suivant leur propre vision.

Bien d'autres aspects présents dans ces journaux, riches de détails, mériteraient d'être explorés : la relation à l'Histoire et aux événements historiques (notamment l'admiration marquée pour Napoléon) ${ }^{139}$, aux arts (peinture et musique : fréquentation assidue des musées, ateliers, concerts, opéras et ballets), les opinions et jugements exprimés vis-à-vis des différents pays visités, souvent mis en parallèle avec la perception du pays natal.

En conclusion de ce parcours, nous aimerions insister sur les contrastes que présente ce vaste ensemble de journaux personnels, et plus précisément sur les accents de modernité qui s'en dégagent : si une part des journaux exprime un point de vue plutôt traditionnel et conventionnel quant au rôle et à la place des femmes au sein de la famille et de la société, une autre part (parfois chez la même diariste) se distingue à la fois par la liberté de ton — souvent moqueur et ironique - , la liberté de mouvement comme de pensée dont témoignent ces textes. Libertés remarquables si on les compare avec les conditions d'existence de leurs contemporaines en France par exemple, à catégorie sociale équivalente : bien peu d'entre elles bénéficient d'un niveau d'éducation et de culture générale d'une qualité comparable ${ }^{140}$; bien peu ont l'occasion d'effectuer des voyages au long cours à travers toute l'Europe ; enfin, à partir de 1804, le Code Civil consacre l'incapacité juridique totale des femmes mariées en France, et donc l'impossibilité de gérer directement leurs propres biens, ce qui n'est pas le cas en Russie. Passant de la tutelle du père à celle du mari, aucune Française ne jouit alors du degré d'indépendance qui même s'il reste relatif - caractérise les femmes de l'aristocratie russe.

138. Voir Gretchanaia, Viollet, éds., « Si tu lis jamais ce journal... ».

139. E. Grečanaja, C. Viollet, « Diaristes et épistolières russes (fin XVIII'-début XXe siècles) : Reflets de l'Histoire », in Nicole Pellegrin, éd., Les voyageurs dans 1'histoire, Presses universitaires de Saint-Étienne (à paraître, 2011).

140. Voir Françoise Mayeur, L'Éducation des filles en France au XIXe siècle, P. : Hachette, 1979. 
Le thème de l'argent n'est nullement tabou, et les diaristes, jeunes ou moins jeunes, semblent être parfaitement au courant de l'état des finances, soit durant le voyage, soit dans la famille de manière générale. Dans son journal, Natal' ja Golicyna relate qu'elle gère elle-même sa propriété en Russie centrale, y aménage les villages en faisant construire maisons et église, en un mot «arrange et règle toutes ses affaires ${ }^{141}$. Ekaterina Barjatinskaja, séparée de facto de son mari, possède une « propriété aux environs de Saint-Petersbourg » ${ }^{142}$ et mène un train de vie indépendant, voyageant souvent seule à travers toute l'Europe. Elizaveta Šahovskaja parle des 200000 mille roubles de dette de sa mère ${ }^{143}$, du montant des émoluments qu'elle verse à la gouvernante de sa fille, etc. Se préparant au mariage, elle réfléchit à la meilleure manière de gérer ses biens : «Je trouve que c'est un crime de négliger l'administration de ses biens, et surtout de la confier à des intendants loués » car, dit le proverbe, c'est « l'œil du maître [qui] engraisse les chevaux »144. Âgée d'une vingtaine d'années, elle prend très au sérieux cette question :

Quand on connoit ses revenus, quand on s'en sert avec modération, on a la jouissance d'être tranquille, de donner une bonne éducation à ses enfants, on n'est pas réduit à leur refuser les maîtres ; on peut même leur donner des talents agréables. On a le plaisir de contenter ses serviteurs, et leur ôter par là le moyen de désobéissance [...]. Depuis qu'on m'a remis les comptes de Spaskoe [sa propriété], je suis très diligente, et je m'occupe de l'ordre, c'est une école pour l'avenir. ${ }^{145}$

Enfin, les relations avec l'autre sexe constituent l'un des objets privilégiés du discours des diaristes. Si, comme on peut s'y attendre, l'élan d'amour envers l'élu de leur cœur emplit des pages et des pages, voir des journaux entiers chez certaines (Elizaveta Šahovskaja, Marija Bahmeteva...), d'autres diaristes font preuve d'une certaine audace en racontant leurs flirts - notamment les joutes verbales, d'un esprit souvent acerbe, qui se déroulent au bal. Les jugements sur l'autre sexe sont souvent sévères, on se moque de certains travers, du manque d'éducation, d'élégance et de raffinement ; on observe de près et juge sans concession la manière dont les hommes mariés se conduisent avec leur épouse. Lorsque le journal de jeune fille se prolonge au-delà du mariage, il n'est pas rare qu'il décrive les tonalités de la vie conjugale, avec ses hauts et ses bas, bonheurs, craintes et déceptions. On trouve même des descriptions assez audacieuses des relations physiques entre époux, comme chez Marija Bahmeteva :

Il m'a commencé d'embrasser, si j'aurai suivi mes sentimens j'aurais fait la même chose, mais j'ai pensé que peut-être je ne le toucherai pas, mais enfin il a été si caressant pour moi que j'ai tout oublié et j'été prête à lui baiser les mains. [...] après peu de tems il m'a appelé dans mon cabinet, m'a commencé

141. Remarques sur mes voyages (1783-1790), $1.4 \mathrm{v}$.

142. Gretchanaia, Viollet, éds., « Si tu lis jamais ce journal... », p. 105.

143. Journal. Commencé à Colp le 15 mars 1832,1. 8v .

144. Journal. 1823, RGB, f. 336/2, carton 47, d. 3, 1. 123.

145. Ibid., 1. $123 \mathrm{v}^{\circ}$. 
d'embrasser et croyoit obtenir ce que l'amour a de plus tendre mais je me suis enfuit chez ma belle mère. ${ }^{146}$

Au cours d'une « conversation très animée avec sa sœur sur le mariage », Elizaveta Šahovskaja affirme qu'elle veut, avant de se marier, prendre tout son temps afin «d'examiner le caractère, l'esprit, la raison» de son futur époux, s'assurer qu' « aucun sacrifice fait pour [elle] ne lui sera pénible », et ne jamais douter que « son cœur sera entier à [elle]». Car, insiste-t-elle : « Ce n'est pas un mari que je veux, non ; c'est un ami, un compagnon pour cette vie, et pour l'autre ${ }^{147}$. Sa fille Natal'ja considère que si «les dames russes sont mieux que les françaises », les Messieurs en revanche, tant français qu'italiens, «sont bien plus libres qu'en Russie », qui, exceptés ceux de sa propre famille, « ont l'air très peu distingués » ${ }^{148}$.

Tenant durant plusieurs années son carnet de bal, Elena Pavlovna Zaharževskaja (née Tiesenhausen, 1804-1890) relate les conversations durant les danses - y compris avec le Tsar - et résume en ces termes humoristiques la (longue) soirée du 12 août 1828 :

Beaucoup dansé, beaucoup causé, beaucoup ri avec le Père la Joie - mon fidèle chevalier Schilling. Les 1ères contredanses avec le gracieux Polonais Vincent d'Abry; les 2des avec Wulf - la mazourka avec Stenbock, mon danseur effectif et avec Schilling, mon danseur postiche - pour la partie causante. Le pot pourri hélas ! avec un Trappiste, je crois car il avait fait vœu de se taire - un aimable inconnu, Mr Silencieux enfin, puisqu'il faut lui donner un nom - Un ennui sans pareil durant une heure et demie ! Ouf ! je n'en pouvais plus. C'était déjà un malheur mit Unglück vermischt [mêlé au malheur]. Après un souper fort gai où j'eus encore le gros Schilling pr voisin et partner, l'on dansa un gros Vater, où Mr Vincent d'Abry se distingua encore par ses sauts et gambades et par sa voix sonore et harmonieuse. ${ }^{149}$

On retrouve le même ton allègre et impertinent chez la jeune Ekaterina Fredericks, qui discute « vengeance et rancune » tout en valsant avec ses partenaires, à qui elle aime jouer des tours et observer l'effet produit, surtout sur celui qu'elle décrit comme «bête comme un corridor sombre ${ }^{150}$, tandis qu'elle se définit elle-même comme « une immense force d'attaque ». Serait-elle inspirée par le cynisme d'un roman comme Les Liaisons dangereuses lorsqu'elle écrit :

Madame, vous ne m'avez pas devinee, vous n'avez pas deviné que votre ambition n'est qu'une naine à coté de la mienne. Je ne peux pas agir par moi-meme aussi je ne peux etre ambitieuse que pour qui j'aime et alors c'est sans limites : Maintenant j'aime Pierre. Eh bien pour lui je suis capable de [...] me marier au

146. Journal depuis le 10 août 1805, par Marie de Bachmeteff, 1. 79.

147. Journal depuis le 22 mai 1822, RGB, f. 336/2, carton 47, d. 2,1. 13v, 19.

148. [Journal, 1841-1842], 1. 38.

149. [Journal/carnets de bal, 1820-1828], RGALI, f. 1337, op. 1, d. 74,1. 20.

150. Gretchanaia, Viollet, éds., « Si tu lis jamais ce journal... », p. 327. 
premier puissant dont il aura besoin — je suis capable de devenir grande dame pour le faire avancer et reussir. ${ }^{151}$

Quant à Matil'da Golynskaja, âgée d'une vingtaine d'années et qui voyage en Europe, elle s'éprend d'un jeune vicomte espagnol rencontré à Naples, avec qui elle passe des moments seule à seul, et qui lui fait des confidences sur sa vie sentimentale : «Comme j'aurais voulu aimer, mais en sorte que cela soit payé de retour», s'exclame-t-elle, « comme j'aurais pu aimer le vicomte si !!!, hélas, son cœur n'était déjà pris... » Elle «meurt d'envie » de lui faire le «shakehand », mais finalement la raison l'emporte, elle se reprend: «Je trouvais que ce n'était pas nécessaire d'être aimable avec un homme engagé - c'est comme s'il était marié ${ }^{152}$. D'autres hommes lui feront ensuite la cour, mais sans jamais constituer un parti acceptable.

Sa sœur Praskov'ja, de deux ans son aînée, tombe amoureuse d'un certain Ledeau : «Je sentis que c'était un homme distingué sous tous rapports, et [...] j'aurais été bien charmée qu'il se plût avec moi ! » ; « je voulais plaire (j'ai honte de l'avouer) parce qu'il me plaisait ; je sentais que je n'hésiterais pas à l'épouser s'il en avait le désir »; mais peu après, elle se morigène pour sa tendance à «s'attacher à tout homme qu'on voit ». Lorsqu'un autre homme, italien cette fois, lui fait la cour, elle lui déclare qu'il ressemble à son premier amour, qu'elle considère comme son idéal, et «tout le temps il ne fut plus question que des originaux et des copies »... Toujours est-il qu'elle aussi «trouve indigne d'être si fort occupée d'un homme qu'on ne pourra jamais épouser hélas ! », et se désespère : «Qu'il est doux d'être aimée par un homme qu'on aime ! Mais pourquoi n'est-ce pas mon cas ? ${ }^{153}$.

Sachant fort bien faire la part des choses entre leurs aspirations, leurs penchants sentimentaux et les convenances, les jeunes diaristes ne manquent cependant pas de jouer de ces contrastes, avec humour souvent, et d'affirmer leur personnalité malgré les intérêts de la famille et en dépit des contraintes du monde aristocratique. Leur liberté de ton, leur sincérité et leur humour inscrivent ces écrits personnels méconnus dans la modernité, à l'égal parfois du célèbre journal de Marija Baškirceva, comme en témoigne par exemple Ekaterina Fredericks :

Mon idée fixe principale (malgré moi car c'est une faiblesse) c'est de parvenir à plaire à quelqu'un qui n'estime que l'entourage.

Une des secondes c'est de tourner autant de têtes que possible pour me faire un grand état-major parce qu'un état-major c'est un joli entourage. ${ }^{154}$

elgretchanaia@rambler.ru

IMLI, Académie des Sciences, Moscou

cviollet@wanadoo.fr

ITEM, CNRS-ENS, Paris

151. [Journal, 1854], GARF, f. 1074, op. 1, d. 426,1. 34v.

152. [Journal, 1846-1847], 1. 12-17.

153. [Journal, 1841-1842], 1. 6-16.

154. [Journal, 1852], GARF, f. 1074, op. 1, d. 426, 1. 18. 